\title{
Plant host shift: The viability and persistence of a
}

\section{polyphagous, non-native insect}

\author{
Isabelle Bueno Silva ${ }^{1}$, Blake McGrane-Corrigan ${ }^{2}$, Oliver Mason ${ }^{2}$, Rafael de Andrade \\ Moral $^{2}$ and Wesley Augusto Conde Godoy ${ }^{1 *}$ \\ ${ }^{1}$ Entomology and Acarology Department- ESALQ, Piracicaba, SP, Brazil \\ ${ }^{2}$ Department of Mathematics and Statistics, Maynooth University, Maynooth, Co. Kildare, Ireland
}

*Corresponding author. Email: wacgodoy@ usp.br; Phone: +55 (19) 3429-4199 
Abstract Drosophila suzukii (Diptera: Drosophilidae) has become a pervasive pest in several countries around the world. Many studies have investigated the preference and attractiveness of potential hosts on this invasive, polyphagous drosophilid. Thus far, no studies have investigated whether a shift of fruit host could affect its ecological viability or spatiotemporal persistence. In this study, we analysed the fecundity and oviposition period jointly with the survival time of $D$. suzukii subject to a shift in host fruit, using a joint modelling method for longitudinal outcomes and time-until-event outcomes. The number of eggs laid by females was higher in raspberry than in strawberry and when setting adults of F1 generation underwent a first host shift. The joint modelling also suggested that insects reared on raspberry survived longer. We then combined experimental results with a twopatch dispersal model to investigate how host shift in a species, that exhibits densitydependent dispersal, may affect its asymptotic dynamics. In line with empirical evidence, we found that a shift in host choice can significantly affect the growth potential and fecundity of a species such as D. suzukii, which ultimately could aid such invasive populations in their ability to persist within a changing environment. 


\section{Introduction}

The adaptation of non-native species to new environments is essential for biological invasion processes, which involve colonization, establishment, and local or regional dispersal events (Hengeveld 1989). Drosophila suzukii (Matsumura 1931) is an example of such an invader species. It originated in Asia and was first observed outside its native range in 1980 in Hawai'i (Kaneshiro, 1983), and then later in Europe (Calabria et al. 2012), the United States (Bolda et al. 2010) and Mexico (Senasica 2013). D. suzukii was first recorded in Brazil in 2013 (Deprá et al. 2014), and then in Argentina, Uruguay, and Chile (Cichón et al. 2015; González et al. 2015; Medina-Muñoz et al. 2015). Compared to other fruit flies, such as Zaprionus indianus (Gupta 1970), D. suzukii has a serrated ovipositor (Atallah et al. 2014), which allows the larvae to develop inside its host fruit. This allows the entry of pathogens that can seriously damage the fruit, resulting in significant losses in crop production (Lee, J.C. et al. 2011a; Walsh et al. 2011; Knapp et al 2020). The morphology and polyphagous behaviour of $D$. suzukii makes it a harmful and pervasive pest of many fruit and berry crops (Lee, J.C. et al. 2011b). Several studies have focused on fruit traits associated with the oviposition choice of D. suzukii (Burrack et al. 2013; Kinjo et al. 2013; Poyet et al. 2015; Olazcuaga et al. 2019) and the physiology of the fruit that attracts this drosophilid (Abraham et al. 2015; Wong et al. 2018). For no-choice assays, Diepenbrock et al (2016) found significant differences in the effects of cultivated hostversus non-cultivated host-shift on the biological characteristics of $D$. suzukii. However, to our knowledge, there is no study that has analysed the impacts of host shift on the invasive Brazilian population. 
Previous experiments designed to investigate host shift in polyphagous pests have proved important when studying the demographic responses of insects facing new hosts (Kennedy and Storer 2000). Within laboratory experiments can be combined with theoretical models of population growth to allow the population dynamics of such organisms to be simulated over many successive generations based on empirical parameter estimates, longer than what is feasible in vivo (Heaps et al. 2016; Dey and Joshi 2018).

In recent years, additional structure has been implicitly and explicitly incorporated into mathematical and computational models, as an attempt to account for the movement of insects at different spatiotemporal scales and life stages (Coutinho et al. 2012; Malaquias et al. 2017). The introduction of spatial structure offers a new perspective when investigating the movement of insects between patches (Okubo and Levin 2001; Moretti et al. 2013), a mechanism that may have significant consequences for integrated pest management and conservation strategies (Nestel 2004). Structural models that have been proposed in the past have tried to model the dynamics of $D$. suzukii by incorporating, for example, probability of occurrence (Ørsted \& Ørsted 2018) and the influence of abiotic factors (Langille et al. 2016; Reyes\& Lira-Noriega 2020; Winkler et al. 2020), however to our knowledge no model has tried to assess the effects of a host-shift on the species' persistence dynamics.

In the present study, we combined laboratory experiments with mathematical modelling to investigate how host shift may affect the local establishment, equilibrium dynamics, and persistence of D. suzukii. This multidisciplinary approach is useful to understand how insects may respond to variations in host availability, which ultimately 
may promote or hinder the ability of such animals to persist in new multi-crop landscapes.

Our null hypotheses were:

- $\quad$ host shift (the change of fruit) does not affect D. suzukii survival and the fecundity viability of immature individuals,

- the macronutrient composition does not change due to host shift, and

- host shift does not affect the equilibrium dynamics and persistence of $D$. suzukii populations.

\section{Methods}

\section{Insect rearing and host-shift assay}

We reared a population of D. suzukii from colonies maintained in the Entomology Laboratory of the Federal University of Pelotas, Rio Grande do Sul State, Brazil. The flies used for the assays were kept in cages $(30 \times 21 \times 17 \mathrm{~cm})$ in climate chambers (Fitotron $®$ ) at $25 \pm 1^{\circ} \mathrm{C}$, relative humidity $70 \pm 5 \%$ and $14-10$ h photoperiod. $D$. suzukii was chosen as a model insect in the present work because it is a continental invasive species of high relevance for fruit growing worldwide and especially in Brazil. The flies received fresh raspberries cultivated without insecticides, obtained from farms in southeastern Brazil, to obtain the first experimental generation. Fruits with eggs were removed and incubated under the same conditions until emergence. Raspberries were chosen as hosts because they are subject to high fruit-fly infestations in Brazil (Klesener et al. 2018).

Flies that emerged in raspberry (generation F1) were placed on either raspberry or strawberry, with five replicates, in a completely randomised design (Figure 1). Each replicate consisted of one test tube $(7.8 \mathrm{~cm}$ height $\times 3.8 \mathrm{~cm}$ diameter $)$ with one strawberry 
or two raspberries and ten couples of $D$. suzukii. After the adults of the next (F2) generation emerged, they were placed in another test tube containing raspberries or a strawberry. This step was repeated, in a completely randomised design, but now with three replicates, each consisting of a test tube with five females and three males of $D$. suzukii from the F2 generation (Figure 1). Then, we repeated the entire assay using the same protocols, with the only difference being the number of replicates for the F2 generation, which was five for the second assay (Figure 1). The fruits were replaced daily, and the number of eggs and daily mortality of adults were recorded over the F1 and F2 generations. We observed the total number of eggs laid in each replicate, egg-adult viability, total oviposition period (in days), and survival time of the adults (in days).

\section{Analysis of macronutrient concentrations}

Three randomly selected adults from each generation (F1, F2 and F3, Figure 1) were placed individually in Eppendorf tubes containing 100\% ethanol. Four macronutrients: protein, lipid, carbohydrate and glycogen were extracted from each fly, following Lorenz's (2003) protocol, and prepared separately for quantification in a spectrophotometer (Epoch Microplate Spectrophotometer, BioTek).

Protein was quantified using $300 \mu \mathrm{L}$ of Coomassie reagent (Coomassie Plus Protein, Pierce Biotechnology, Inc., Rockford, IL, USA), and $10 \mu \mathrm{L}$ of each sample, in triplicate. Five standard curves were prepared, based on concentrations from $0 \mu \mathrm{g} / \mathrm{mL}$ to $2000 \mu \mathrm{g} / \mathrm{mL}$, following the manufacturer's protocol. The absorbance used was $595 \mathrm{~nm}$. For lipid quantification, we used vanillin as the reagent in a sulfuric acid solution, and 10 $\mu \mathrm{L}$ of each sample, in triplicate. We used commercial vegetable oil to obtain the five 
standard curves (concentrations of $0 \mathrm{mg} / \mathrm{dL}$ to $2500 \mathrm{mg} / \mathrm{dL}$ ), at an absorbance of $525 \mathrm{~nm}$

(Van Handel 1985). Carbohydrates and glycogen were quantified using anthrone reagent (Roe 1955). For the carbohydrate aliquots, we prepared a $50 \mu \mathrm{L}$ solution of each sample individually, containing $100 \mu \mathrm{L}$ of sulfuric acid and $200 \mu \mathrm{L}$ of anthrone. The glycogen aliquots were prepared with a solution containing $25 \mu \mathrm{L}$ of the sample, $50 \mu \mathrm{L}$ of sulfuric acid and $100 \mu \mathrm{L}$ of anthrone. The five standard curves were constructed, with glucose used as default (concentrations of $0 \mathrm{mg} / \mathrm{dL}$ to $100 \mathrm{mg} / \mathrm{L}$ ) and an absorbance of $620 \mathrm{~nm}$.

\section{Statistical analyses}

Quasi-Poisson models (Demétrio et al. 2014) were fitted to the data for total number of eggs, including the effects of assay (to reflect the experimental design), generation, host, and the interaction between generation and host in the linear predictor, and the natural logarithm of the number of females as an offset term. F tests were performed to assess the significance of the effects, and multiple comparisons were performed by obtaining the $95 \%$ confidence intervals for the linear predictors. Goodness-of-fit was assessed using halfnormal plots with simulation envelopes (Moral et al. 2017).

To study the association between oviposition behaviour over time and survival times of the insects, a joint model for longitudinal outcomes (oviposition) and time-untilevent outcomes (survival times) was fitted to the data (Rizopoulos 2010). For the longitudinal part of the model, we log-transformed the count data and included the effects of the assay (to reflect the experimental design), generation, host, time, all two-way interactions between generation, host, and time, and the three-way interaction amongst these factors in the linear predictor as fixed effects. We also included a random intercept 
per female, given that the number of eggs laid by the same female is correlated over time. For the survival part of the model, we used a Cox proportional-hazards model and included the effects of the assay, generation, and host, and the interaction between generation and host in the linear predictor. This modelling strategy allows for the estimation of an association parameter, which in this study measures the effect of fecundity on the mortality risk (Rizopoulos 2010). We performed likelihood-ratio (LR) tests to assess the significance of the fixed effects for both parts of the model and a Wald test to assess the significance of the association parameter.

The quantitative data for metabolised macronutrients were analysed by fitting linear models including the effect of treatment (F1 raspberry, F2 raspberry, F3 raspberry, and F3 strawberry) in the linear predictor. The significance of the treatment effect was assessed using $\mathrm{F}$ tests.

\section{The model}

The experimental results described above illustrate the impacts that nutritional value have on local growth, as well as the possible effects that shifting between hosts has on overall population levels. We will now describe a two-patch mathematical model that exhibits qualitatively similar behaviour over a longer timescale.

It has been observed that dispersal is, in general, an asymmetric process and may have significant consequences for population persistence (Rinnan 2018), especially when assessing the impacts of climate change and monitoring species range shifts (Zhou and Kot 2011). When introducing explicit spatiality into population models, a phenomenon known as Turing instability may arise, whereby the extinction equilibrium in the absence of 
dispersal is asymptotically stable, but by allowing dispersal it becomes unstable (Neubert et al. 2002). Thus, including spatial structure in models could provide more qualitative insights into the dynamics of populations.
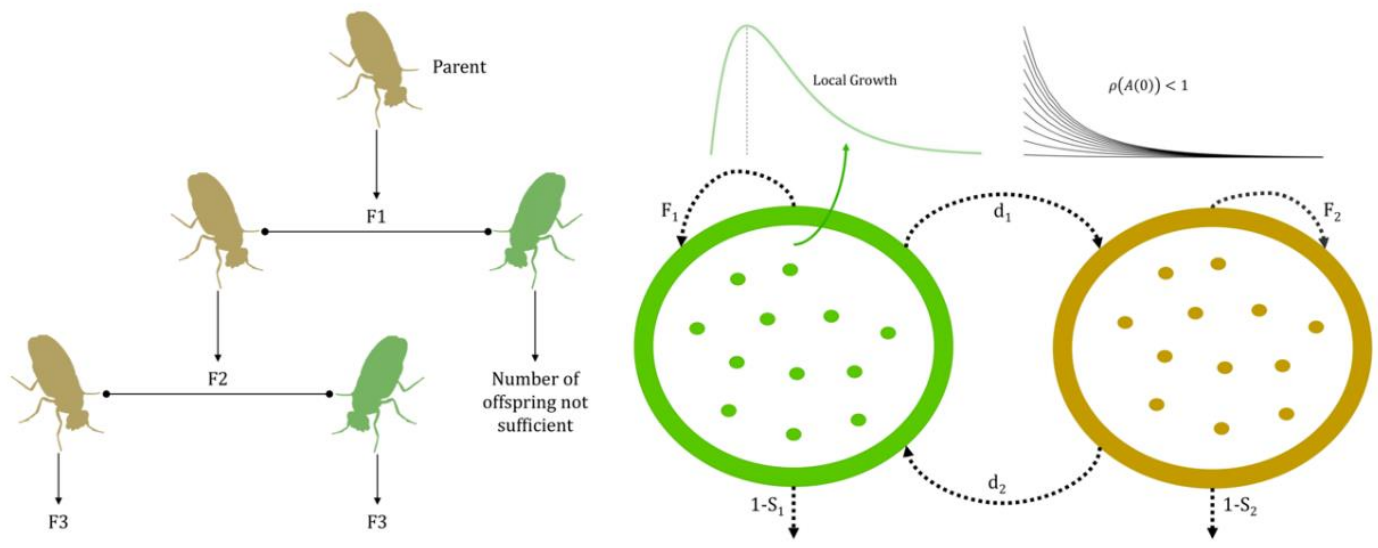

Figure 1. Host-shift assay (left) showing the experimental design performed. Raspberry was the parental generation and subsequent filial generations where reared on both raspberry and strawberry. Population dynamics within and between patches (right), governed by both demographic and dispersal processes. When dispersal is not allowed the local dynamics is driven

Consider a polyphagous, single-species population that inhabits two nonidentical patches, and denote the patch population densities, in generation $t \in \mathbb{Z}_{\geq 0}$, by $x_{1}(t)$ and $x_{2}(t)$, respectively. Following reproduction on a patch we assume both subpopulations disperse between patches, which depends continuously on their respective densities. We also assume that the overall population is spatially closed, i.e. individuals reproduce and disperse only on the two specified patches. This may not be ecologically realistic, but our aim is to understand the potential effects of dispersal between two differing patches on the 
overall population dynamics. A general form for such a model is given by the system of difference equations

$$
\begin{aligned}
& \mathrm{x}_{1}(\mathrm{t}+1)=\left[1-\mathrm{d}_{1}\left(\mathrm{x}_{1}(\mathrm{t})\right)\right] g_{1}\left(\mathrm{x}_{1}(\mathrm{t})\right) \mathrm{x}_{1}(\mathrm{t})+\mathrm{d}_{2}\left(\mathrm{x}_{2}(\mathrm{t})\right) g_{2}\left(\mathrm{x}_{2}(\mathrm{t})\right) \mathrm{x}_{2}(\mathrm{t}) \\
& \mathrm{x}_{2}(\mathrm{t}+1)=\left[1-\mathrm{d}_{2}\left(\mathrm{x}_{2}(\mathrm{t})\right)\right] g_{2}\left(\mathrm{x}_{2}(\mathrm{t})\right) \mathrm{x}_{2}(\mathrm{t})+\mathrm{d}_{1}\left(\mathrm{x}_{1}(\mathrm{t})\right) g_{1}\left(\mathrm{x}_{1}(\mathrm{t})\right) \mathrm{x}_{1}(\mathrm{t})
\end{aligned}
$$

This formulation considers two continuously differentiable functions, $g_{i}: \mathbb{R}_{+} \rightarrow \mathbb{R}_{+}$and $d_{i}: \mathbb{R}_{+} \rightarrow(0,1)$, that respectively describe local growth and density-dependent dispersal. Dispersal is assumed to be both asymmetric, $d_{i} \neq d_{j}$ for $i \neq j$, and bidirectional, $d_{i} \notin\{0,1\}$. Later, we will briefly discuss the case when dispersal is not permitted in either direction, i.e. each patch is isolated. In the context of $D$. suzukii one can consider subscripts 1 and 2 as representing dispersal from strawberry to raspberry and raspberry to strawberry, respectively. We can rewrite (1) in nonlinear matrix model form

$$
\boldsymbol{x}(t+1)=\boldsymbol{A}(\boldsymbol{x}(t)) \boldsymbol{x}(t), \boldsymbol{x}(0)=x_{0}
$$

where $\boldsymbol{x}=\left(x_{1} x_{2}\right)$ is a state vector in $\mathbb{R}_{+}^{2}=\left\{\boldsymbol{x} \in \mathbb{R}^{2}: \boldsymbol{x} \geq \mathbf{0}\right\}$, the cone of 2-dimensional real vectors with nonnegative components, and $\boldsymbol{A}: \mathbb{R}_{+}^{2} \rightarrow \mathbb{R}_{+}^{2 x 2}$ is a nonnegative projection matrix (Cushing 1998). Our population projection matrix is thus given by

$$
\boldsymbol{A}(\boldsymbol{x})=\left(\begin{array}{cc}
{\left[1-d_{1}\left(x_{1}\right)\right] g_{1}\left(x_{1}\right)} & d_{2}\left(x_{2}\right) g_{2}\left(x_{2}\right) \\
d_{1}\left(x_{1}\right) g_{1}\left(x_{1}\right) & {\left[1-d_{2}\left(x_{2}\right)\right] g_{2}\left(x_{2}\right)}
\end{array}\right)
$$

Typically, matrix projection models are often considered when populations show a clear stage or age structure (Smith and Thieme, 2011), like for example when using Leslie matrix models (Leslie 1945), but we will use them to partition our population into two subpopulations, each with its own vital rates. 


\section{Experimental results}

\section{Total fecundity, oviposition period and proportion of viable eggs}

The daily oviposition rates per female over time are shown in Figure 2. The interaction between generation and host significantly affected the total number of eggs $\left(F_{1,32}=11.05\right.$, $\mathrm{p}=0.0023$ ). The number of eggs laid by females was higher in raspberry than in strawberry, for F1 and F2 generations; and the number of eggs laid in strawberry was higher in generation F2 than in F1 (Table 1). When setting adults of F1 generation in first host shift (Figure 1), available eggs were insufficient to have adults and to set up the second hostshift, as performed with raspberry. The interaction between host and generation did not significantly affect the total oviposition period $(L R=0.54, p=0.4606)$. The global test for risk proportionality suggested that the model well fitted the data $\left(\chi^{2}\right.$ test statistic $=$ 3.16, d.f. $=4, p=0.5316$ ). Thus, analysing the marginal means indicated that the mean period of oviposition did not differ between generations ( $L R=0.10$, d.f. $=1, p=0.7473$ ), but did differ between hosts $(\mathrm{LR}=31.77$, d.f. $=1, \mathrm{p}<0.0001)$. Hence, females spent more time ovipositing in raspberry than in strawberry (Table S1 in Supporting Information).

The interaction between generation and host did not significantly affect the proportion of offspring that reached the adult stage $\left(F_{1,18}=0.07, \mathrm{p}=0.8004\right)$. However, there were significant main effects of host $\left(F_{1,19}=6.88, \mathrm{p}=0.0172\right)$ and generation $\left(F_{1,20}=\right.$ $25.77, \mathrm{p}<0.0001$ ). Even though more eggs were laid in raspberry than in strawberry (see Table 1), the offspring were significantly more viable in strawberry (Table S2 in Supporting Information). 
Table 1. Mean fecundity \pm standard error (number of eggs laid per female), for each host and generation. Different upper-case letters denote significant differences in the rows; different lower-case letters denote significant differences in the columns.

\begin{tabular}{ccc}
\hline & \multicolumn{2}{c}{ Host } \\
\cline { 2 - 3 } Generation & Raspberry & Strawberry \\
\hline F1 & $67.95 \pm 7.70 \mathrm{aA}$ & $0.20 \pm 0.15 \mathrm{bB}$ \\
& & \\
\hline F2 & $50.40 \pm 6.44 \mathrm{aA}$ & $12.98 \pm 11.03 \mathrm{aB}$ \\
\hline
\end{tabular}

\section{Fecundity - survival time relationship and analysis of macronutrients}

The results from the joint modelling of longitudinal oviposition and survival time indicated no significant effect of the three-way interaction between time, generation, and host ( $\mathrm{LR}=$ 0.81 , d.f. $=1, p=0.3678$, nor of the two-way interactions between generation and time $(L R=1.54$, d.f. $=1, p=0.2145)$ or host and time $(L R=2.4$, d.f. $=1, p=0.1210)$. However, the interaction between generation and host was significant $(\mathrm{LR}=6.53$, d.f. $=1, \mathrm{p}=$ 0.0106), as was the main effect of time (Wald test $p<0.0001$ ). Since the time estimate was negative $(-0.0197$ with a standard error of 0.0043$)$, we concluded that the oviposition rate decreased over time. For the survival part of the model, the interaction between generation and host was not significant $(\mathrm{LR}=0.17$, d.f. $=1, \mathrm{p}=0.6777)$, nor was the main effect of generation $(\mathrm{LR}=1.04$, d.f. $=1, \mathrm{p}=0.3073)$. However, the main effect of host was significant $(L R=6.82$, d.f. $=1, p=0.0090)$, which suggests that insects reared on raspberry 
survived longer. We conclude that high fecundity is associated with a lower mortality risk, i.e., a longer survival time on the fruit, given the negative estimate of the association parameter $(-0.5608$ with a standard error of 0.1476 , Wald test $\mathrm{p}<0.0001)$. The analysis of macronutrients shows us concentrations of lipid $\left(\mathrm{F}_{3,8}=1.11, \mathrm{p}=0.4010\right)$, protein $\left(F_{3,8}=\right.$ $2.32, \mathrm{p}=0.1520)$, carbohydrate $\left(F_{3,8}=0.19, \mathrm{p}=0.8950\right)$, and glycogen $\left(F_{3,8}=0.51, \mathrm{p}=\right.$ 0.6880) did not differ significantly amongst individuals of the F1, F2, and F3 generations (Table S3 in Supporting Information).

\section{Stability and persistence results}

\section{Definitions and local dynamics}

Before analysing model (2), with $\boldsymbol{A}(\boldsymbol{x})$ given by (3), we will first recall some key mathematical definitions needed for the results described later in this section. For more detail on these and related results on nonlinear dynamical systems, matrix theory and persistence theory, the reader should consult the Supporting Information.

An equilibrium or fixed point $\boldsymbol{x}^{\star}$ of (2) is a solution of $\boldsymbol{x}=\boldsymbol{A}(\boldsymbol{x}) \boldsymbol{x}$. The equilibrium $\boldsymbol{x}^{\star}$ is said to be stable if for any $\epsilon>0$ there exists a $\delta>0$ such that $\left|\boldsymbol{x}_{0}-\boldsymbol{x}^{\star}\right|<\delta$ implies that $\left|x(\mathrm{t})-\boldsymbol{x}^{\star}\right|<\epsilon$ for all $\mathrm{t}$, where $\boldsymbol{x}_{0}$ denotes the initial condition $\boldsymbol{x}(0)$. If, in addition, there is some $R>0$, such that $\boldsymbol{x}(t) \rightarrow \boldsymbol{x}^{\star}$ as $t \rightarrow \infty$ for any solution with $\left|\boldsymbol{x}_{0}\right|<R$, the equilibrium is locally asymptotically stable. If this holds for any $R>0$, it is said to be globally asymptotically stable. Clearly, any nonlinear matrix model has an equilibrium at the origin, i.e. the extinction equilibrium. If the extinction equilibrium is (locally or globally) asymptotically stable, then $\lim _{t \rightarrow \infty} x(t)=\mathbf{0}$, i.e. the state vector asymptotically 
tends toward the origin. The mathematical definitions of persistence, which we will now recall, characterise the opposite situation, where populations are maintained at some positive level asymptotically.

Given a general nonlinear matrix model of the form (2) with state space $\mathbb{R}_{+}^{n}$ we say that the system is uniformly weakly persistent with respect to a persistence function $\eta: \mathbb{R}_{+}^{n} \rightarrow[0, \infty)$ if there exists some $\epsilon>0$, such that for each solution $\boldsymbol{x}(t)=\left(x_{1}(t) x_{2}(t) \cdots x_{n}(t)\right)$, with initial condition $\boldsymbol{x}(0)=\boldsymbol{x}_{0} \in \mathbb{R}_{+}^{n}$ and $\eta\left(\boldsymbol{x}_{0}\right)>0$,

$$
\limsup _{t \rightarrow \infty} \eta(x(t))>\epsilon
$$

The persistence function is typically some quantitative measure of population abundance, such as the total or site-specific population size for example. Intuitively, weak uniform persistence means that for any time $T>0$, there is some $t>T$ at which the persistence measure exceeds $\epsilon$. A stronger concept of persistence is that of uniform strong persistence, which is identical to the above definition apart from replacing the limsup with the liminf. Strong uniform persistence means that there is some $T>0$ such that the persistence function exceeds $\epsilon$ for all later times $t>T$. 


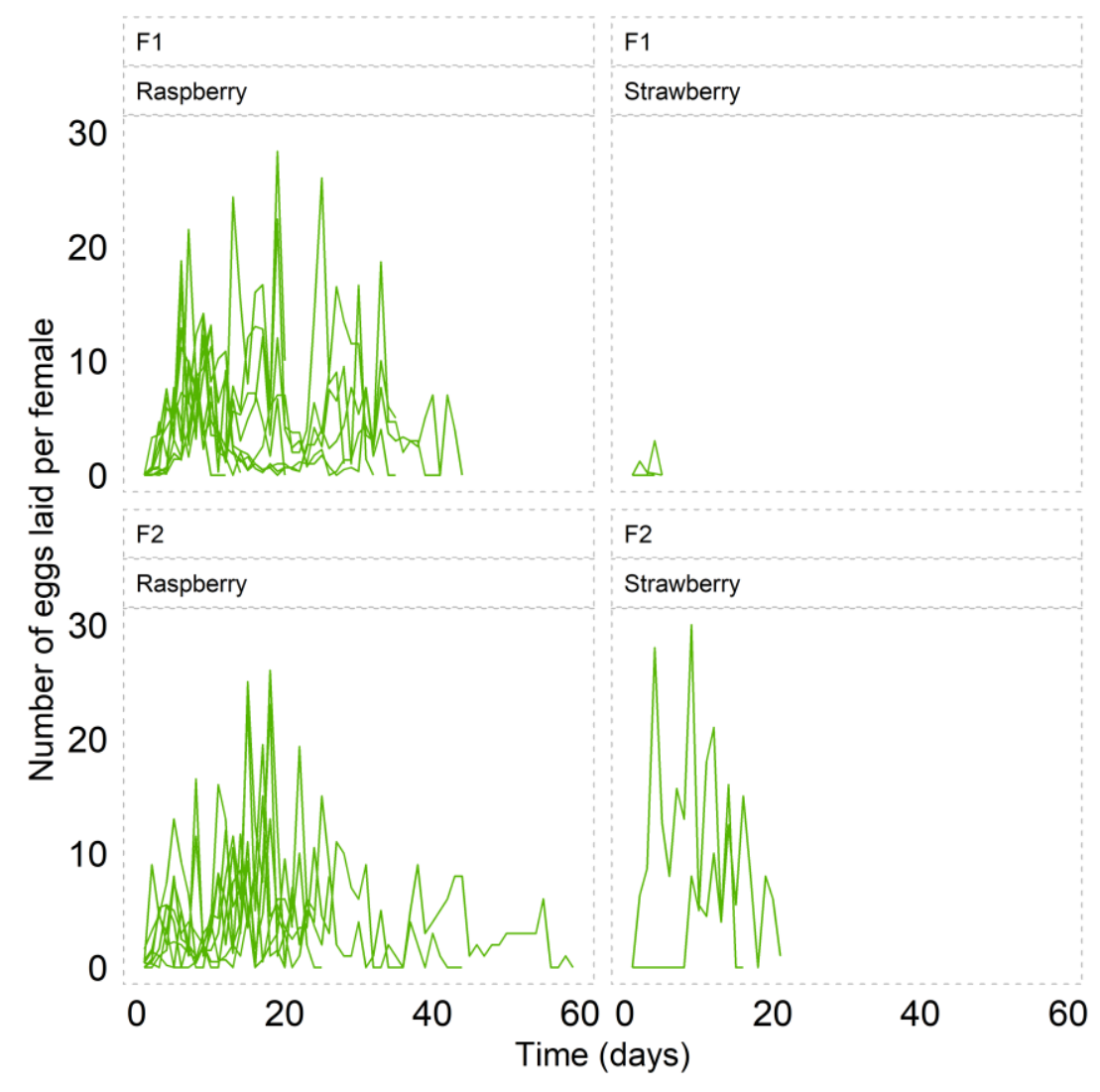

Figure 2. Number of eggs laid per female, in the F1 (A and B) and F2 (C and D) generations. Each line represents one replicate, which included ten and five females for generations F1 and F2, respectively.

We assume that the local growth function takes the form

$$
g_{i}\left(x_{i}\right) \triangleq R_{i} F_{i} S_{i} e^{-\alpha x_{i}}
$$

for $i=1,2$, also known as the recruitment or fitness function (Chesson 2012; Eager at al. 2012). Parameters $F_{i}>0, S_{i} \in(0,1), R_{i} \in(0,1)$ can be interpreted as respectively being the fertility, survival probability and sex ratio on patch $i$. Density-dependence is assumed to affect each local population in the same way, with $\alpha>0$ being the influence of intraspecific competition for resources. Note that the difference in each patch is determined by 
the effects of nutrition and fecundity, as expressed through $S_{i}$ and $F_{i}$ being different for each patch. This follows from our experimental findings that the interaction strength between each population and their host was greatly influenced by the number of eggs laid and the nutrition source. These factors ultimately determine if each generation successfully reproduce and survive to the next generation.

Recruitment function (4) was inspired by Prout and McChesney (1985), who used a difference equation to describe intraspecific competition within an immature $D$. melanogaster population and ultimately how this affected adult fertility and survival. They assumed egg survival and fertility were decreasing functions of adult density. They also included a sex ratio of a half. In our model we use constant demographic and dispersal parameters. When the two patches are isolated, the dynamics on patch $i$ is given by

$$
x_{i}(t+1)=g_{i}\left(x_{i}(t)\right) x_{i}(t) \triangleq f_{i}\left(x_{i}(t)\right)
$$

This is similar in form to a Ricker map (Ricker 1954). It is unimodal, meaning it attains a unique highest value or global maximum. This occurs at $x_{i}=\alpha^{-1}$. The maximum possible population size is then $g_{i}\left(\alpha^{-1}\right) \alpha^{-1}=R_{i} F_{i} S_{i}(e \alpha)^{-1}$ and so the population is bounded from reaching arbitrarily large values. It can also be seen from (4) and (5) that if $R_{i} F_{i} S_{i}<1$, for $i=1,2$, then $x_{i}(t) \rightarrow 0$ as $t \rightarrow \infty$. The unique nontrivial equilibrium for patch $i$, $\hat{x}_{i}=\ln \left(R_{i} F_{i} S_{i}\right)(\alpha)^{-1}>0$ if, and only if, $R_{i} F_{i} S_{i}>1 . f_{i}$ is continuously differentiable at $\hat{x}_{i}$, which means the local stability properties of this equilibrium can be determined by the linear approximation $x_{i}(t+1)=\hat{x}_{i}+f_{i}^{\prime}\left(\hat{x}_{i}\right)\left(x_{i}(t)-\hat{x}_{i}\right)$, provided the difference $x_{i}(t)-\hat{x}_{i}$ is sufficiently small (Elaydi, 2005). Local asymptotic stability is guaranteed 
once $\left|f_{i}^{\prime}\left(\hat{x}_{i}\right)\right|<1$. From the form of $f_{i}$ we can see that $\hat{x}_{i}$ is locally asymptotically stable precisely when $\left|f_{i}^{\prime}\left(\hat{x}_{i}\right)\right|=\left|1-\ln \left(R_{i} F_{i} S_{i}\right)\right|<1 \Leftrightarrow 1<R_{i} F_{i} S_{i}<e^{2}$.
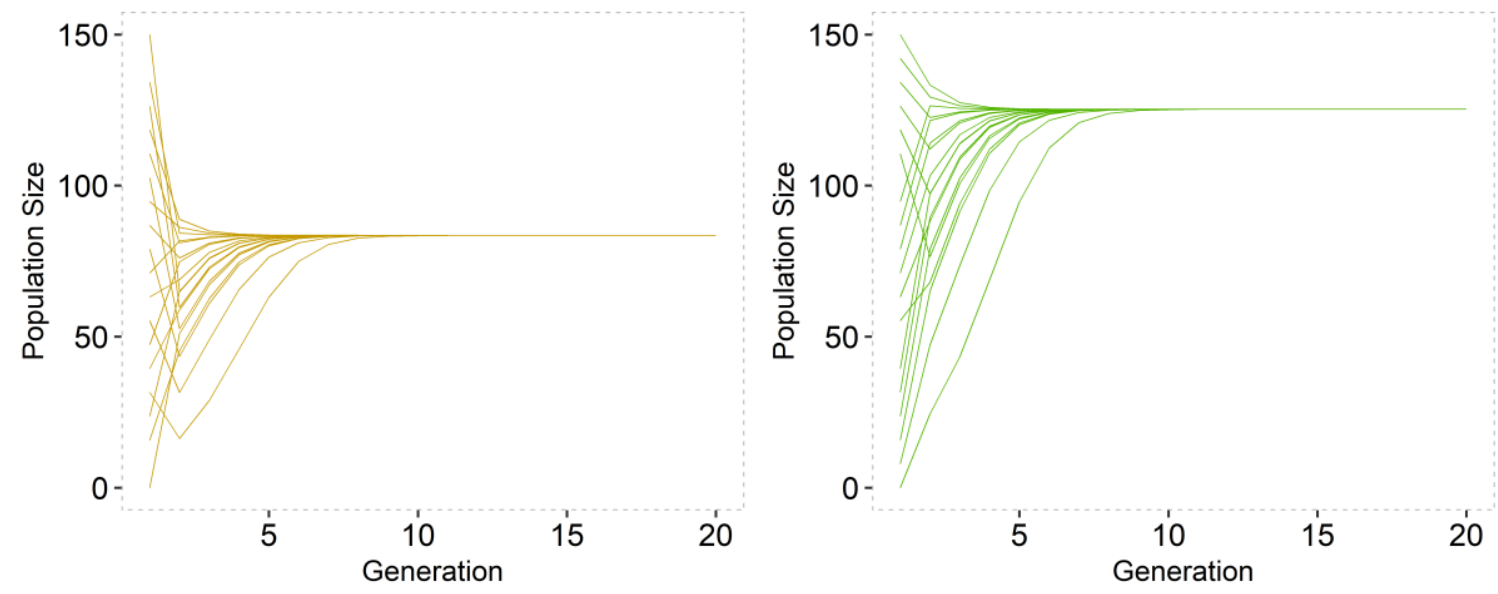

Figure 3. Patch trajectories for strawberry (left) and raspberry (right) with $R_{i} F_{i} S_{i}$ given by empirical estimates obtained from experiments and passive dispersal parameters $d_{1}=0.6$ and $d_{2}=0.4$. They approach stable positive equilibria for all initial conditions between 1 and 150 .

\section{Density-dependent dispersal}

We will now consider model (1) rewritten as a nonlinear matrix model (2), with local patch dynamics given by (4). We assume that dispersal is bidirectional, asymmetric and a density-dependent process. In the rest of the paper, $\rho(\boldsymbol{B})$ denotes the spectral radius of a square matrix $\boldsymbol{B}$. We refer the reader to the Supporting Information for formal definitions and proofs of the results presented below. 
As a first example, let us look at the scenario when we have passive/constant dispersal, i.e. $d_{i}\left(x_{i}\right) \equiv p_{i} \in(0,1)$ for $x_{i} \geq 0$. As $e^{-\alpha x_{i}} \leq 1$ for $x_{i} \geq 0, i=1,2$, it can be seen that $\boldsymbol{A}(\boldsymbol{x}) \leq \boldsymbol{A}(\mathbf{0})$ for all $\boldsymbol{x} \geq 0$ in $\mathbb{R}^{2}$. It now follows that for any $\boldsymbol{x}(0) \in \mathbb{R}_{+}^{2}$,

$$
x(t)=A(x(t-1)) A(x(t-2)) \cdots A(x(1)) A(x(0)) x(0) \leq A(0){ }^{t} x(0)
$$

and so, if $\rho(\boldsymbol{A}(\mathbf{0}))<1$, then $\boldsymbol{A}(\mathbf{0})^{t} \rightarrow 0$ as $t \rightarrow \infty$. Therefore, the extinction equilibrium is globally asymptotically stable. This gives a sufficient condition for ensuring that we have patch extinction, as is shown in Smith and Thieme (2011) for general nonlinear matrix models.

In the context of pest management, we are primarily interested in how the presence/absence of dispersal influences persistence over time and what drives the population on either patch to extinction (eradication). We are also interested in knowing if on either patch, both assumed to differ in nutritional quality, the subpopulations can persist even at low densities. This is a major concern in agricultural management, namely small numbers of individuals persisting on ephemeral low-quality habitat and then exhibiting rapid growth when favourable conditions arise (Reigada et al. 2018).

It is well known that for any induced matrix norm $|\cdot|$, the spectral radius of $\boldsymbol{B}$ satisfies $\rho(\boldsymbol{B}) \leq|\boldsymbol{B}|$ (Horn and Johnson, 2012). As the $l_{1}$ induced matrix norm is given by the maximum of the column sums of a matrix, it follows that $\rho(\mathbf{A}(\mathbf{0}))<1$ if all column sums of $\mathbf{A}(\mathbf{0})$ are less than 1 . This implies that a sufficient condition for $\rho(\mathbf{A}(\mathbf{0}))<1$ is $\mathrm{R}_{\mathrm{i}} \mathrm{F}_{\mathrm{i}} \mathrm{S}_{\mathrm{i}}<1$. This could be interpreted as each patch population having either low survival probability and fertility, high fertility but low survival probability or vice versa, which intuitively will lead to the eventual decline of the population in the long run. 
For the remainder of this section, we consider what happens when $\rho(\boldsymbol{A}(\mathbf{0}))>1$, so local asymptotic stability of the extinction equilibrium is not guaranteed. The results presented below, will show that dispersal can lead to patch persistence when its absence could result in extinction on one patch. This is akin to dispersal driven growth or what is known as Turing instability (Neubert at al. 2002; Guiver et al. 2017), whereby allowing dispersal prevents a previously declining and isolated population from going extinct, a somewhat similar concept to the so-called rescue effect in metapopulation theory (Hanski 2008).

We will first note that $F(\boldsymbol{x}) \triangleq \mathbf{A}(\boldsymbol{x}) \boldsymbol{x}$, with $\mathbf{A}(\boldsymbol{x})$ given by (3) and local patch dynamics given by (4), is strongly positive.

Lemma 1. Let $\boldsymbol{A}(\boldsymbol{x})$ be given by (3) and local patch dynamics given by (4), with $d_{i}\left(x_{i}\right) \in(0,1) \alpha>0, F_{i}>0, S_{i} \in(0,1)$ and $R_{i} \in(0,1)$, for $i=1,2$. Then for any $\boldsymbol{x} \in \mathbb{R}_{+}^{2} \backslash\{\boldsymbol{0}\}, F(\boldsymbol{x}) \gg \mathbf{0}$.

Lemma 1 also implies that $\mathbb{R}_{+}^{2} \backslash\{\boldsymbol{0}\}$ is forward invariant under $F$, i.e., trajectories that begin inside the positive orthant remain there for all time. In our next result, we note that when the spectral radius of $\boldsymbol{A}(\mathbf{0})$ is greater than 1 there exists a positive patch-coexistence equilibrium of (2). Here, $\boldsymbol{x} \gg \mathbf{0}$ means that all components of the vector $\boldsymbol{x}$ are positive.

Proposition 1. Let $\boldsymbol{A}(\boldsymbol{x})$ be given by (3) and local patch dynamics given by (4), with $d_{i}\left(x_{i}\right) \in(0,1), \alpha>0, F_{i}>0, S_{i} \in(0,1)$ and $R_{i} \in(0,1)$, for $i=1,2$.

Suppose that $\rho(\boldsymbol{A}(\mathbf{0}))>1$. Then there exists some $\boldsymbol{x} \gg \mathbf{0}$ with $F(\boldsymbol{x})=\boldsymbol{x}$. 
In the following lemma, we show that for $\boldsymbol{A}(\boldsymbol{x})$ be given by (3) and local patch dynamics given by (4), the system (2) is point dissipative. This simple fact is needed for the results on persistence that follow.

Lemma 2. Let $\boldsymbol{A}(\boldsymbol{x})$ be given by (3) and local patch dynamics given by (4), with $d_{i}\left(x_{i}\right) \in(0,1), \alpha>0, F_{i}>0, S_{i} \in(0,1)$ and $R_{i} \in(0,1)$, for $i=1,2$. Then there exists some $M>0$ such that for any $\boldsymbol{x}(0) \in \mathbb{R}_{+}^{2},|\boldsymbol{x}(t)|_{1} \leq M$ for all $t \geq 1$.

The previous lemma is needed to establish the first of our main results.

Theorem 1. Let $\boldsymbol{A}(\boldsymbol{x})$ be given by (3) and local patch dynamics given by (4), with $d_{i}\left(x_{i}\right) \in(0,1), \alpha>0, F_{i}>0, S_{i} \in(0,1)$ and $R_{i} \in(0,1)$, for $i=1,2$. Suppose that $\rho(\boldsymbol{A}(\mathbf{0}))>1$. Then, there exists some $\epsilon>0$ such that

$$
\liminf _{t \rightarrow \infty}|\boldsymbol{x}(t)|_{1}>\epsilon
$$

for any solution with $\boldsymbol{x}(0) \in \mathbb{R}_{+}^{2} \backslash\{\mathbf{0}\}$.

This means that when $\rho(\boldsymbol{A}(\mathbf{0}))>1$ system (2), with projection matrix given by (3) and local patch dynamics given by (4), is uniformly strongly persistent with respect to the total population size across the two patches. We will next show that a stronger result than Theorem 1 is possible, as $F(\boldsymbol{x})$ is strongly positive. 
Theorem 2. Let $\boldsymbol{A}(\boldsymbol{x})$ be given by (3) where $F_{i}>0, S_{i} \in(0,1), R_{i} \in(0,1)$, $\alpha>0$ and $d_{i}\left(x_{i}\right) \in(0,1)$ for $i=1,2$. Let $\tilde{\eta}(\boldsymbol{x})=\min \left\{x_{1}, x_{2}\right\}$. Suppose that $\rho(\boldsymbol{A}(\mathbf{0}))>1$. Then, there exists some $\epsilon>0$ such that

$$
\liminf _{t \rightarrow \infty} \tilde{\eta}(x(t))>\epsilon
$$

for any solution with $\boldsymbol{x}(0) \in \mathbb{R}_{+}^{2} \backslash\{\mathbf{0}\}$.

As a second example, let us look at the scenario when $R_{1} F_{1} S_{1}<1$ and $R_{2} F_{2} S_{2}>1$, so on patch 1 the population tends to extinction and on patch 2 there is high survival and fertility. For $d_{i}(0) \in(0,1)$ we have that

$$
\boldsymbol{A}(\mathbf{0})=\left(\begin{array}{cc}
{\left[1-d_{1}(0)\right] R_{1} F_{1} S_{1}} & d_{2}(0) R_{2} F_{2} S_{2} \\
d_{1}(0) R_{1} F_{1} S_{1} & {\left[1-d_{2}(0)\right] R_{2} F_{2} S_{2}}
\end{array}\right)
$$

and so, for $R_{2} F_{2} S_{2}$ sufficiently large, one can ensure that both uniform strong persistence and patch-persistence occur. For example, this can be easily seen if we let $R_{1} F_{1} S_{1}=0.9, R_{2} F_{2} S_{2}=50, d_{1}=0.5$ and $d_{2}=0.7$, meaning that $\rho(\boldsymbol{A}(\mathbf{0})) \approx 16.01>$ 1. By Theorem 2, this implies that, even though on one patch extinction may be inevitable, by allowing dispersal one can not only rescue the declining patch from extinction and help the overall population to recover, but also ensure that each individual patch population also persists.

Locally a sink population may become extinct in the absence of immigration or source patches, but dispersal can allow the overall population the opportunity to persist. For fast-growing species with short life cycles and for those that exhibit irruptive dynamics, like for example insect species, populations can reach (and exceed) their carrying capacity very quickly, likely leading to high intraspecific competition and density dependent 
dispersal (Powell and Bentz 2014; Goodsman et al. 2017). Low quality habitat (sinks) that are distributed among higher quality habitat (sources) can limit the movement of organisms in space, so identifying the mechanisms that limit growth can vary between habitat types (Heinrichs et al. 2016). Our simple modelling approach suggests that dispersal and demographic effects are potential drivers of population persistence of $D$. suzukii. It is for this reason that modelling population dynamics within a spatial framework can provide new qualitative insights into the mechanisms that drive source-sink dynamics.
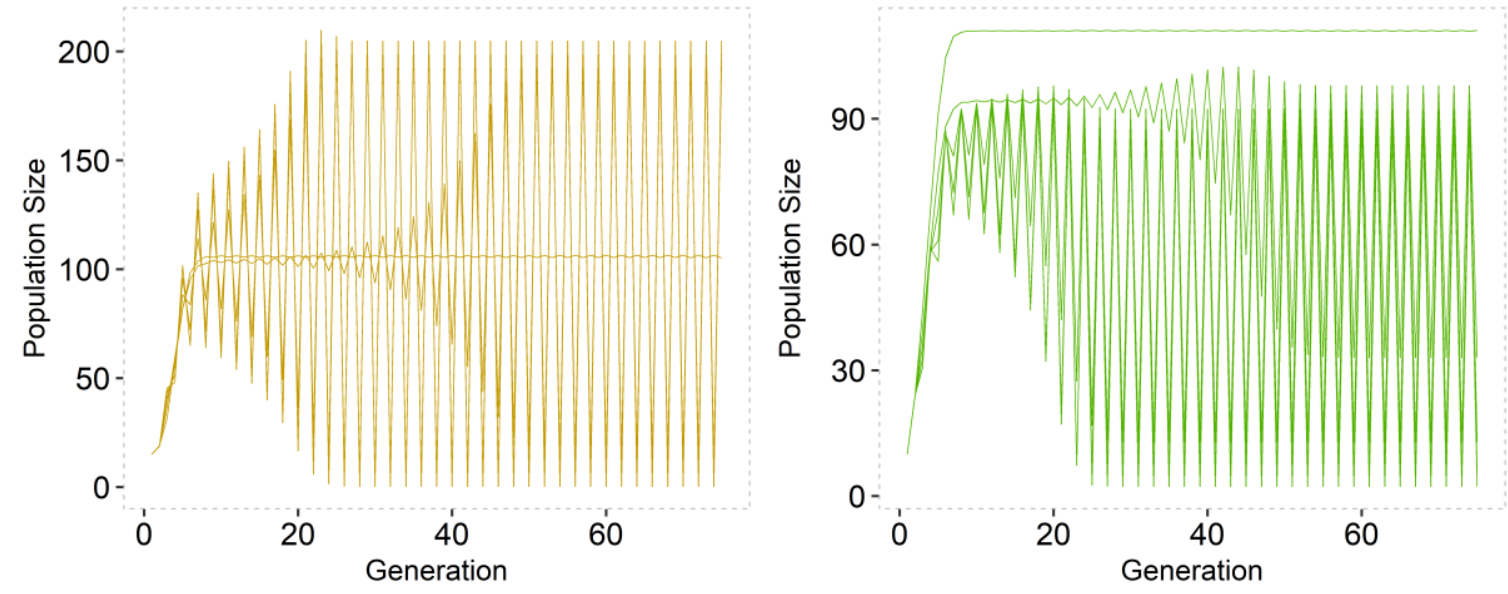

Figure 4. Boost-bust type dynamics for both patch populations, where $R_{i} F_{i} S_{i}$ are given by empirical estimates and $\mu_{i}$ ranging from 0.01 to 0.05 . Initial conditions were $(10,10)$. After initially increasing, trajectories either spproach stable equilibria or oscillate indefinitely.

\section{Bifurcation diagrams and numerical simulations}

For the purpose of simulating model (1), with $g_{i}\left(x_{i}\right)$ given by (4), we must specify a form for $d_{i}\left(x_{i}\right)$, as this is an arbitrary function taking values in $(0,1)$. We will therefore let density-dependent dispersal from patch $i$ be given by

$$
d_{i}\left(x_{i}\right)=1-r_{i} e^{-\mu_{i} x_{i}}
$$


Here $\mu_{i}$ is the strength of density dependence dispersal and $r_{i} \in(0,1)$ is a scaling factor that can be interpreted as the minimum amount of dispersal that is allowed. If we let the number of individuals that remain on patch $i$ be Poisson distributed with mean $\mu_{i}$ then $e^{-\mu_{i}}$ is the probability of obtaining a zero, i.e. the probability of no dispersal. Hence $1-e^{-\mu_{i} x_{i}}$ may be interpreted as the probability of dispersing from patch $i$, which decreases faster with a growing patch density. An estimate for $\mu_{i}$ was obtained from Prout and McChesney (1985), who used a density dependent term of 0.0064 (the sum of $\mathrm{f}$ and $\mathrm{s}$, the sensitivity parameters for fertility and survival, respectively) in their demographic model. We also set $r_{1}=r_{2}=0.9$, which corresponds to permitting a minimum of $10 \%$ dispersal between both patches.
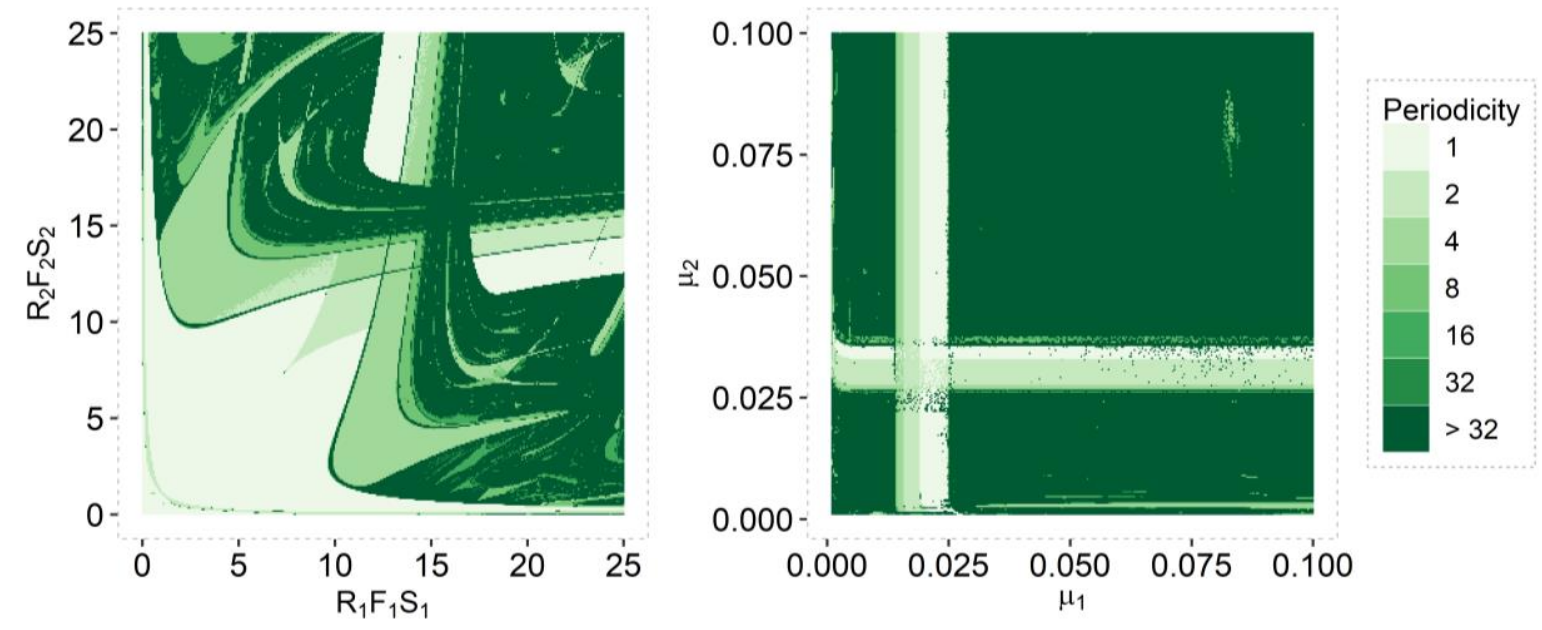

Figure 5. Bifurcation diagrams for the parameter product $R_{i} S_{i} F_{i}$ (left) and $\mu_{i}$ (right) for $i=1,2$, showing the number of unique population sizes according to the colour gradient (stability for values of 1 and periodic orbits for higher values than 1). We considered the last 100 observations after iterating our model for 1000 generations. Initial conditions where $(10,10)$ for both patches. Note that we only show bifurcation diagrams for patch 1 as the diagrams are identical for patch 2 . 
Table 2. Demographic and dispersal parameters

\begin{tabular}{ccccccccc}
\hline Parameter & $\mathrm{R}_{1}$ & $\mathrm{~S}_{1}$ & $\mathrm{~F}_{1}$ & $\mathrm{R}_{2}$ & $\mathrm{~S}_{2}$ & $\mathrm{~F}_{2}$ & $\alpha$ & $r_{i}$ \\
\hline Estimate & 0.5 & 0.335 & 9.43 & 0.5 & 0.216 & 21.7 & 0.0064 & 0.9 \\
\hline
\end{tabular}

For the dispersal function given by (5) and local dynamics given by (4), we simulated our model for 75 generations, for various combination of $\mu_{i}$ and using the experimentally derived demographic parameters (see Figure 4). These parameter values are given in Table 2. We assumed a sex ratio of a half, as this accurately reflects the ratio in the models of Prout and McChesney (1985). We can see that after initial increases in both patch densities, trajectories either settle on a stable equilibrium or oscillate around this equilibrium indefinitely. We extended the total number of generations to 500 and these oscillations did not cease but increased in intensity. Even though both patch populations exhibited oscillatory dynamics, they seem to persist in the long run and although trajectories approach 0 , and then increase rapidly to high densities, these boom-bust type dynamics are common in insect population models (Goodsman, Cook \& Lewis, 2017; Strayer et al., 2017).

Different methods have been proposed to analyze the sensitivity of parameters in population growth models. Among the approaches used for sensitivity analysis, bifurcation analysis stands out. Bifurcation analysis aims to evaluate the stability properties of steady states under parameter variation. This is particularly useful for investigating the association of ecological patterns of oscillation in populations with changing values of demographic 
parameters. This approach allows one to quantify the contribution from changes in parameter values to changes in model outputs (Van Voorn and Kooi 2017).

We conducted a bifurcation analysis to test our model's stability in the $R_{i} F_{i} S_{i}$ and $\mu_{i}$ parameter spaces (see Figure 5). Bifurcation diagrams emerge from relationships between parameter values and population sizes (Dercole \& Rinaldi 2012). Usually, the parametric space on the one axis determines significant changes on the other axis, expressing long-term population behaviour. As a result of this relationship, it is possible to observe stable trajectories, limit cycles with fixed maximum and minimum limiting values and chaos, a regime characterized by total unpredictability, that is, by the absolute absence of fixed cycles (Luque et al. 2011).

We chose to use the product of the demographic parameters in our bifurcation analysis as they play an important role in determining conditions for persistence and the presence of a positive equilibrium. For the $R_{i} F_{i} S_{i}$ bifurcation diagram we set $\mu_{1}=0.2$ and $\mu_{2}=0.3$, to reflect low-moderate density dependence (see Figure 5). We simulated trajectories for 4 different points that correspond to varying limit cycle periodicities (see Figure 6). In Figure 6 we see that at low values of $R_{i} S_{i} F_{i}$ we have stabile equilibria. As we allow demographic parameters to reach values roughly above 10, trajectories are quite unpredictable, in that the period of these limit cycles begin to become greater than 32 . Many discrete models exhibit similar complex dynamic behaviour like that seen in Figure 6 (Hassell, Comins \& May, 1991; Geritz and Kisdi, 2004). Trajectories may seem predictable for some parameter ranges, either stabilising or fluctuating after initial increases, but outside these ranges deterministic chaos is generally observed. Note that the empirical parameter estimates give $R_{i} F_{i} S_{i}$ within the stable region of the parameter space. 


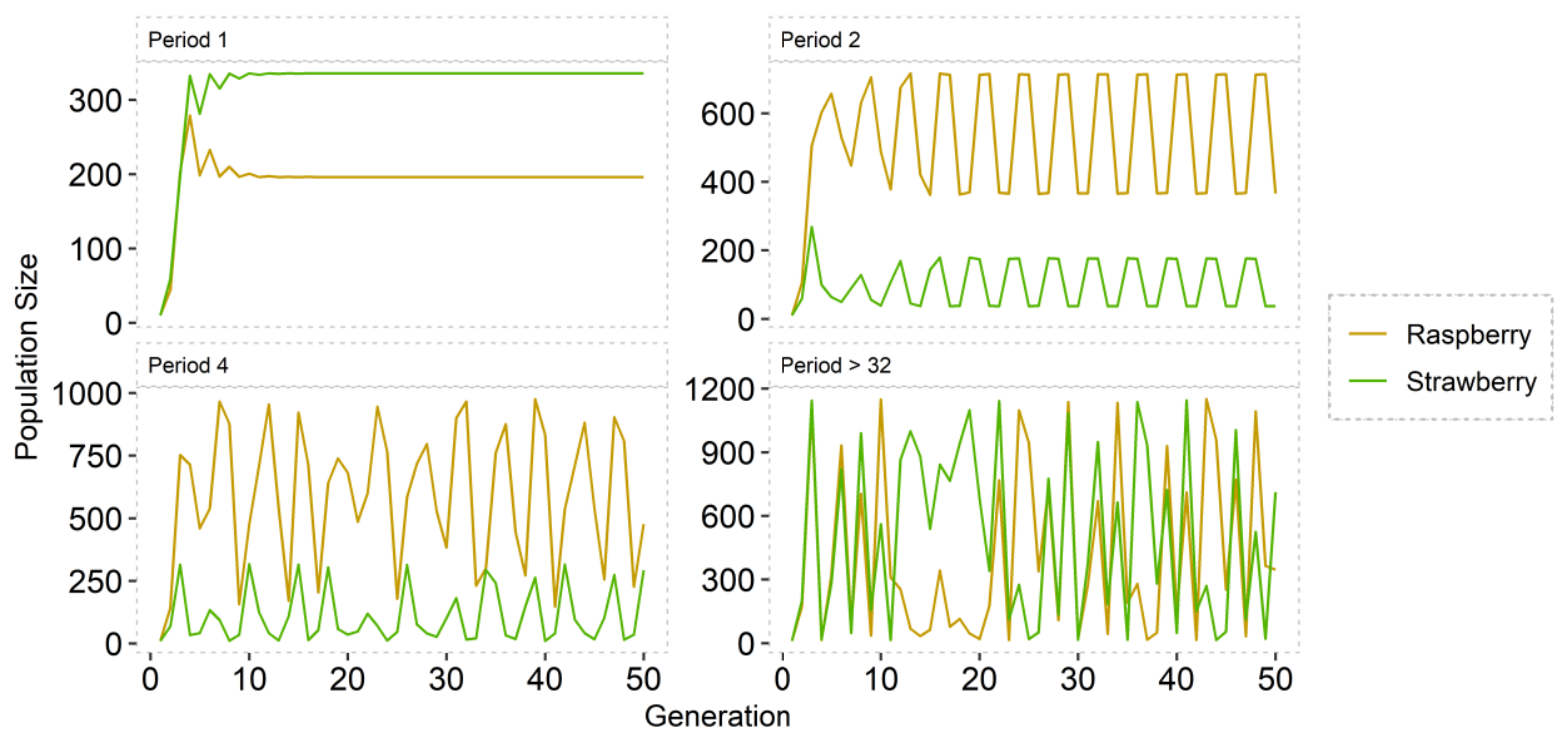

Figure 6. Patch dynamics of strawberry (yellow) and raspberry (green) patches with initial conditions (10, 10), showing the influence of the $R_{i} S_{i} F_{i}$ parameter space with $\mu_{1}=0.2$ and $\mu_{2}=0.3$. Periods refer to the period of the limit cycles.

For the $\mu_{i}$ bifurcation diagram we set $R_{1} F_{1} S_{1}=20$ and $R_{2} F_{2} S_{2}=24$, as the products obtained from empirical estimates showed stable trajectories for all values of $\mu_{i}$. Thus, we were interested to explore what happens when both patches exhibit high survival and fertility. The narrow range within the $\left(\mu_{1}, \mu_{2}\right)$ parameter space (see Figure 5), where trajectories seem to approach a stable fixed point (the uniqueness of which is undetermined), show how sensitive density-dependent dispersal can be. As we increase both parameter values, more complex dynamical behaviour occurs, which may allude to increased density dependent effects on individuals who remain on each patch. This may be because $R_{i} F_{i} S_{i} \gg 0$ (for $i=1,2$ ), meaning subpopulations have higher survival probabilities or increased fertility on their respective patches which can lead to increasing competitive interactions. Each patch also has a nonzero influx/outflow of individuals 
to/from it, and this may permit sufficient genetic mixing, with dispersal being one of the main drivers of genetic variation in insect species (Raymond et al. 2013).

The demographic parameters considered in Table 2 determine the conditions for the existence of a patch coexistence equilibrium. For values of $\mu_{i}$ within the narrow band in Figure 6 one can observe that we always have $\rho(\boldsymbol{A}(\mathbf{0}))>1$. Proposition 2 then implies that we have existence of a positive equilibrium and by Theorems 1 and 2 we have persistence. We have not mathematically explored the stability and uniqueness of this fixed point but instead have derived conditions for our population projection matrix that ensures we have both uniform strong persistence and patch-persistence.

\section{Discussion}

No-choice and choice tests are frequently used to evaluate the host and/or prey range of insects (Faria et al. 1999; Van Driesche \& Murray 2004; Diepenbrock et al. 2016). Studies comparing choice with no-choice conditions have found that insects undergo important changes in behaviour, mainly in new areas they occupy. In our experiments, the results of the no-choice change of fruits showed that the quality of the food source impacted fecundity, period of oviposition and proportion of viable populations for D. suzukii, thus rejecting hypotheses 1 and 2 . When exploiting resources in crop edges with different host plants, insects in extensive monocultures have little resource choice. In these circumstances, knowledge of the performance and adaptive potential of invading species is desirable, because successful invaders can become major pests at high densities. 
The response of the biological parameters to this host shift showed that disturbances mainly affect the proportion of nutrients necessary to allow immature individuals to develop, as already shown for other Drosophila species (Lee, K.P. et al. 2008; Matavelli et al., 2015; Rodrigues et al. 2015). The change in this nutrient proportion, even evaluated indirectly as in the present study, helps to understand the changes in ecological viability of a species.

In the present study, the high number of eggs of D. suzukii found in the second generation, for insects reared on strawberry, may reflect their parental nutritional conditions, as insects of one generation that feed on good-quality host plants are likely to successfully produce subsequent filial generations (Matzkin et al. 2013). Even though the control group, i.e., (raspberry), produced a high number of eggs, the egg-adult proportion was high in the group fed on strawberry. This could be explained by both the high number of eggs in food with good nutritional quality, which can increase competition for resources for example (Hardin et al. 2015) and, in the group impacted by a change in food quality, the high viability seems to indicate maximum resource use of the offspring, i.e., a tradeoff. In adverse environmental conditions, the reproductive cost to individuals may change to compensate for impaired fitness, resulting in trade-offs between reproduction investment and adult survival (Stearns 1989). For D. suzukii, strategies involving offspring number and viability in conditions of host shift have not been previously described.

The negative impact of biological parameters could not be explained by the intake of macronutrients, not rejecting hypothesis 2 . A possible explanation is the diversity of the microbiota associated with these insects, since symbiotic bacteria can help their insect host to assimilate nutrients (Shin et al. 2011; Sommer \& Newell 2018; Su et al. 2013). The 
diversity of symbionts can drastically affect assimilation of nutrients, which changes with environmental conditions and with corresponding changes to the host food source (Obadia et al. 2018; Yun et al. 2014; Guidolin \& Cônsoli 2017), even at optimal nutrient ingestion. Therefore, the development of polyphagous insects may be effected by different factors acting together, including endosymbiosis, as shown for D. melanogaster (Shin et al. 2011) and D. suzukii (Bing et al. 2018).

The results for the joint modelling for D. suzukii, considering the association parameter, indicate that fecundity can be related to the risk of mortality. Interest in joint models has recently increased, especially for studying the association between longitudinal and time-to-event processes (Alsefri et al. 2020). This approach makes it possible to analyse the risks involved in an interacting/feedback system, particularly the relationship between fecundity and population mortality, as in the current study.

The present study was conducted on an ecological temporal scale, and the data on the influence of host-shift were evaluated over two generations. The dispersal model employed in this study arose from a classical metapopulation perspective, proposed to analyse the dispersal of insects between finitely many patches (Hanski \& Gaggiotti 2004, Moretti et al. 2013, Dey \& Joshi 2018). Mathematical models of population growth coupled by migration or dispersal have often been used to analyse population stability by employing the use of bifurcation diagrams (Ruxton 1996; Moretti et al. 2013; Pal et al. 2018). Values of demographic parameters and dispersal rates generally have a strong association with the stability of population equilibria (Moretti et al. 2013; Pal et al. 2018; Cheng et al. 2019).

Dispersal has been suggested as a possible stabilising mechanism for populations that inhabit local sinks, through what has been termed dispersal driven growth (DDG) 
(Guiver et al. 2017). Our model results further support this hypothesis for spatially structured populations, by showing that dispersal may rescue a species from extinction, even when fertility and survival proportions are low on one of the patches, i.e., one of subpopulations have low local fitness. In model simulations we found that, including density dependent dispersal and intraspecific competition, trajectories exhibit a range of behaviour and are highly sensitive to changes in parameter values. It must be kept in mind that these simulations excluded demographic or environmental stochasticity and so although our final deterministic model 'skeleton' seems to approach a positive fixed point after some time, these somewhat smooth dynamics might oscillate significantly, and may even get arbitrarily close to the basin of attraction of the extinction equilibrium (Hastings et al. 2021).

In the context of pest control the opposite of DDG is usually desired (Mazzi and Dorn 2012), and so our results show that the disruption of common dispersal events could be a mechanism to prevent long term population persistence. DDG is an important means for preventing extinction, especially for populations that are dispersing in the absence of external immigration, from matrix habitat or marginal source patches, like for example in agricultural landscapes (Duelli and Obrist 2003). Ephemeral patch dynamics, whereby patches are sequentially restored and destroyed, play a significant role in allowing pest populations that undergo pulsed dispersal to persist (Reigada et al. 2015). Even in the presence of control strategies, such as the use of pesticides or the introduction of natural enemies, pests may exhibit what is referred to as the hydra effect, whereby increasing their mortality rate results in a positive contribution to population growth (McIntire and Juliano 2018; Costa and dos Anjos 2018). We have not considered habitat destruction or inter- 
species interactions. Extending our work to incorporate these scenarios may prove important, along with increasing the number of patches above the simplest case of two.

Knowing the potential consequences that host shift and biocontrol have on the persistence of crop pests is then vital for food security and for understanding mechanisms of controlling invasions (Bernal and Medina 2018). By including spatial structure in models of herbivore pest dynamics, managers may have the opportunity to determine what series of patches contribute to overall decline/growth of a population (James et al. 2015). Not only does this afford one to know the direct effects of dispersal on a focal host plant, but it also could help one understand the indirect role that pest control could have on adjacent natural matrix habitats (Rand, Tylianakis and Tscharntke 2006). Our models demonstrate that in the absence of dispersal, and under certain parameter constraints, subpopulations on both patches may go extinct. But when connectivity between patches is allowed, we have shown that both uniform strong persistence and patch-persistence can arise, given that the spectral radius of $\boldsymbol{A}(\mathbf{0})$ is greater than unity. When this occurs, we have also found the existence of a positive steady state. The stability properties of this fixed point are not known but simulations suggest that populations can either asymptotically approach this (seemingly unique) fixed point, following transient oscillations around it, or oscillate around it indefinitely. For brevity we have excluded an analysis to determine equilibria stability, as persistence is known as a more robust condition that is commonly used to understand a model's qualitative properties (Jansen and Sigmund, 1998). Ultimately our models suggest that density-dependent dispersal is a vital mechanism by which populations can persevere in a spatial context and inhibiting dispersal, even between 
two patches (the simplest spatial case), could have significant consequences for the longevity of populations in a patchy landscape.

The bifurcation analysis performed in this study showed regions of stability and instability in the parametric spaces of both the demographic and dispersal parameters. The limit of the values investigated in the bifurcation diagrams are compatible with real values obtained in our experiments. The instability of both populations of $D$. suzukii in general can tend to occur with higher values of the parameters, with exceptions. Narrow ranges that allow for stable trajectories show the importance of parametrising models with experimental data. Among the factors capable of changing the population stability in drosophilids, density, competition, food quality, fecundity and migration stand out (Tung et al. 2018), as demonstrated using our theoretical and empirical findings.

Studies that emphasise the relationship of demographic parameters with the stability of equilibria has long been discussed (Ruxton 1996; Moretti et al. 2013; Pal et al. 2018; Cheng et al. 2019). There are compensatory mechanisms that can explain the relationship between life history and the demographic parameters involved in the growth rate and carrying capacity, which suggests possible compensations for populations in low and high densities, as already investigated in D. melanogaster (Mueller \& Ayala 1981).

The availability of food resources can determine the formation of high densities per unit of food substrate in insects and consequently trigger intraspecific competition for food (Hardin et al. 2015), which negatively influences the magnitude of demographic parameters associated with population growth and equilibrium (Klomp 1964; Johst; Berryman \& Lima 2008). High survival and fecundity values are generally associated with limit cycles and can even produce deterministic chaos (Johst, Berryman \& Lima 2008). 
Polyphagous insects such as $D$. suzukii can resort to fruit exchange because of the fly density on the fruit that has been initially exploited (Hardin et al. 2015; Olazcuaga et al. 2019) and the changing conditions of a food source can thus enhance competition between flies on a per-patch level.

As revised in Dey \& Joshi (2018), migration associated with food source can also interfere with the stability of a population. They tested different quantities of adults migrating between patches and concluded that the effect on persistence and constancy of the population can be altered by quality of food and immigration rate between patches. Considered in an applied scenario, the movement of pests between different patches can result in damaging economic losses. In pest control programs the aim is to present actions that maintain the density of pests in an equilibrium level below damaging economic thresholds (Zhang et al 2018; Wieser et al. 2019; Bryant et al. 2020), so the analysis of parameter values that lead to the loss of stability, resulting in unpredictable population outbreaks, has an unquestionable utility for integrated pest management strategies (Costa \& Faria 2010). The ecological interactions between different species of drosophilids and their varied food sources have been systematically studied in the past (Buruga \& Olembo 1971), due to the importance of these species in attacking ripening fruit. Considering the modified structure of the ovipositor of D. suzukii females (Atallah et al. 2014) and the expression of genes associated with chemoreceptors (Crava et al. 2016), the strong response to the food source (fruit host in particular) becomes apparent. The ability of $D$. suzukii to pierce the integument of still-healthy fruits makes it a harmful pest, and therefore, plant host-shift is highly important in commercial fruit growing. The empirical and theoretical results obtained in this study furthers the understanding of host-shift, it's 
possible ecological mechanism and biological consequences for D. suzukii, in particular when different host fruits are available. Another possible extension to the model proposed in this paper could be to include stage structure, somewhat like the infamous LPA model (Dennis et al. 2001), in order to deduce what developmental stages affect host shift and population growth.

\section{Acknowledgements}

We thank Dr Daniel Bernardi for kindly providing the first individuals of Drosophila suzukii to establish our populations. We thank Ms Felipe Andreazza and Dr Eugênio Eduardo de Oliveira for valuable discussions on the subject, Dr Carolina Reigada for her assistance with the experimental design, and Dr Fernando Luis Cônsoli for aid in the macronutrient analyses. The authors thank Dr Janet W. Reid for revising some of the English text. We also thank the Conselho Nacional de Desenvolvimento Científico e Tecnológico (CNPq) for granting a Master's scholarship to IBS. WACG is supported by a research fellowship from CNPq. BMC is supported by the Irish Research Council funded Government of Ireland Postgraduate Scholarship GOIPG/2020/939.

\section{Disclosure}

The authors declare that they have no conflict of interest. 


\section{Supporting Information for}

\section{Plant host shift: The viability and persistence of a}

\section{polyphagous, non-native insect}

\section{Appendix 1 \\ Preliminaries}

For $n \in \mathbb{N}$ with $n \geq 2$ denote by $\mathbb{R}^{n \times n}$ the space of all real square matrices of dimension $\mathrm{n}$, and by $\mathbb{R}^{n}$ the usual n-dimensional real Euclidean space.

Given an $n \times n$ matrix $\boldsymbol{A} \in \mathbb{R}^{n \times n}$, the spectrum of $\boldsymbol{A}, \sigma(\boldsymbol{A})$, is the set of all eigenvalues of

$\boldsymbol{A}$. The spectral radius of $\boldsymbol{A}$ is defined as

$$
\rho(\boldsymbol{A}) \triangleq \max \{|\lambda|: \lambda \in \sigma(\boldsymbol{A})\}
$$

Given a function $F(\boldsymbol{x})=\boldsymbol{A}(\boldsymbol{x}) \boldsymbol{x}$, for $\boldsymbol{A}: \mathbb{R}^{n} \rightarrow \mathbb{R}^{n \times n}$ and $\boldsymbol{x} \geq 0$, we denote the Jacobian matrix of $F(\boldsymbol{x})$ evaluated at $\boldsymbol{a}$ as $\boldsymbol{F}^{\prime}(\boldsymbol{a})$.

For $\boldsymbol{x} \in \mathbb{R}^{n}$ and $p \geq 1$ we define the $l_{p}$ vector norm as 


$$
|x|_{p} \triangleq\left(\sum_{1}^{n}\left|x_{i}^{p}\right|\right)^{\frac{1}{p}}
$$

and the matrix norm induced by the $l_{p}$ norm as

$$
|\boldsymbol{A}|_{p} \triangleq \sup _{|x|_{p}=1}\left\{|\boldsymbol{A} \boldsymbol{x}|_{p}\right\}
$$

We mainly work with the $l_{1}$ norm here. We use the same notation $|\cdot|$ for both the vector and matrix norms. This should not cause confusion as we use bold lowercase letters for vectors and bold uppercase letters for matrices.

A system of the form (2) is said to be point-dissipative or ultimately bounded if there is some $M>0$ such that for all $\boldsymbol{x}(0) \in \mathbb{R}_{+}^{2}$, there exists some $T>0$ such that $|x(t)| \leq M$ for all $t \geq T$.

Denote by $F^{m}=F \circ F \circ \cdots \circ F$ (m times) the m-fold composition of $F: \mathbb{R}^{n} \rightarrow \mathbb{R}^{n}$ with itself, for $m$ a positive integer.

A function $F: \mathbb{R}_{+}^{n} \rightarrow \mathbb{R}_{+}^{n}$ is said to be strongly positive if for all $c>0$ there exists some positive integer $t>0$ such that $F^{t}(\boldsymbol{x}) \gg \mathbf{0}$ for all $\boldsymbol{x} \in \mathbb{R}_{+}^{n}$ with $0<|\boldsymbol{x}(t)| \leq c$ (Smith and Thieme, 2011). 
A matrix $\boldsymbol{B} \in \mathbb{R}^{n \times n}$ is said to be irreducible if there is no proper subset $J \subset\{1, \ldots n\}$ for which $b_{i j}=0$ for all $i \in J, j \in\{1, \ldots, n\} \bigvee$.

Let $\boldsymbol{A} \in \mathbb{R}^{n \times n}$ be nonnegative. Then $\rho(\boldsymbol{A}) \in \sigma(\boldsymbol{A})$ and there exists a vector $\boldsymbol{x} \in \mathbb{R}^{n}$, $\boldsymbol{x} \neq \mathbf{0}$ such that $\boldsymbol{A} \boldsymbol{x}=\rho(\boldsymbol{A}) \boldsymbol{x}$ (Horn and Johnson, 2012). It can then be seen that, for $\mathrm{n} \geq 2, \rho(\boldsymbol{A})<1 \Leftrightarrow \exists$ some vector $\mathbf{v} \gg 0$ in $\mathbb{R}^{n}$ such that $\boldsymbol{A} \boldsymbol{v} \ll \boldsymbol{v}$.

We will finally recall some results from Smith and Thieme (2011) that are relevant in our proofs. Unless stated otherwise, for the rest of this appendix

$$
\boldsymbol{x}(t+1)=\boldsymbol{F}(\boldsymbol{x}(t)), \boldsymbol{x}(0)=\boldsymbol{x}_{0}
$$

for $\boldsymbol{x}=\left(x_{1} x_{2} \cdots x_{n}\right) \in \mathbb{R}_{+}^{n}, \boldsymbol{A}: \mathbb{R}_{+}^{n} \rightarrow \mathbb{R}_{+}^{n x n}$ and $F: \mathbb{R}_{+}^{n} \rightarrow \mathbb{R}_{+}^{n}$ a nonlinear matrix function such that $F(\boldsymbol{x})=\boldsymbol{A}(\boldsymbol{x}) \boldsymbol{x}$.

Theorem A1 (Theorem 7.5 in (Smith and Thieme (2011)): Let $F: \mathbb{R}_{+}^{n} \rightarrow \mathbb{R}_{+}^{n}$ be continuous and assume that there exists $R>0, \boldsymbol{y} \in \mathbb{R}_{+}^{n}$ and some nonnegative matrix $\mathbf{D}$ such that $\rho(\boldsymbol{D})<1$ and

$$
F(x) \leq y+D x
$$

for $\boldsymbol{x} \in \mathbb{R}_{+}^{n}$ and $|\boldsymbol{x}| \geq R$. Let $F$ be differentiable at $\mathbf{0}$ and $\boldsymbol{F}^{\prime}(\mathbf{0})$ be irreducible or $F(\boldsymbol{x})=A(\boldsymbol{x}) x$ with $\boldsymbol{A}(\boldsymbol{x})$ nonnegative, and $\rho\left(\boldsymbol{F}^{\prime}(\mathbf{0})\right)>1$. Then there exists some $\boldsymbol{x} \neq \mathbf{0}$ such that $F(\boldsymbol{x})=\boldsymbol{x}$. 
Theorem A2 (Theorem 7.9 in (Smith and Thieme (2011)): Let $F: \mathbb{R}_{+}^{n} \rightarrow \mathbb{R}_{+}^{n}$ be a differentiable mapping. Suppose that:

1. $\mathbb{R}_{+}^{n} \backslash\{\mathbf{0}\}$ is forward invariant under $\mathrm{F}$.

2. There exists $r_{0}>1$ and $\boldsymbol{v} \gg \mathbf{0}$ such that $\boldsymbol{A}_{0}^{\mathrm{T}} \boldsymbol{v} \geq r_{0} \boldsymbol{v}$, where $\boldsymbol{A}_{0}=\boldsymbol{F}^{\prime}(\mathbf{0})$ and $\boldsymbol{A}_{\mathbf{0}}^{T}$ denotes its transpose.

3. The system $(\dagger)$ defined by $F$ is point-dissipative.

Then system $(\dagger)$ is uniformly $\eta$-persistent for $\eta(\boldsymbol{x})=|\boldsymbol{x}|$ where $|\cdot|$ is any norm on $\mathbb{R}_{+}^{n}$.

In the following result, we use the notation $x\left(t, x_{0}\right)$ to denote the solution of the system $(\dagger)$ at time $t$ corresponding to the initial condition $\boldsymbol{x}_{0}$.

Theorem A3 (Theorem 7.15 in (Smith and Thieme (2011)): Suppose 1-3 from Theorem A2 hold, and $\mathrm{F}$ is strongly positive. Let $\hat{\eta}(\boldsymbol{x})=\min _{i} x_{i}$. Define

$$
X_{0} \triangleq\left\{\boldsymbol{x}_{0} \in \mathbb{R}_{+}^{n}: \hat{\eta}\left(\boldsymbol{x}\left(t, \boldsymbol{x}_{0}\right)\right)=0, t=0,1,2, \cdots\right\}
$$

Then there exists some $\epsilon>0$ such that

$$
\liminf _{t \rightarrow \infty} \hat{\eta}(x(t)) \geq \epsilon
$$

for any solution of (†) with $\boldsymbol{x}(0) \in \mathbb{R}_{+}^{n} \backslash X_{0}$. 


\section{Appendix 2 \\ Proofs}

Proof of Lemma 1: This is trivial as $\boldsymbol{A}(\boldsymbol{x})$ is a positive matrix for all $\boldsymbol{x} \geq \mathbf{0}$.

Proof of Proposition 1: First note that for $x \geq 0$

$$
F(\boldsymbol{x})=\left(\begin{array}{l}
{\left[1-d_{1}\left(x_{1}\right)\right] R_{1} F_{1} S_{1} e^{-\alpha x_{1}} x_{1}+d_{2}\left(x_{2}\right) R_{2} F_{2} S_{2} e^{-\alpha x_{2}} x_{2}} \\
d_{1}\left(x_{1}\right) R_{1} F_{1} S_{1} e^{-\alpha x_{1}} x_{1}+\left[1-d_{2}\left(x_{2}\right)\right] R_{2} F_{2} S_{2} e^{-\alpha x_{2}} x_{2}
\end{array}\right)
$$

It can be seen that $x_{i} e^{-\alpha x_{i}} \leq(\alpha e)^{-1}$ for $x_{i} \geq 0, i=1,2$, it is easy to see that there exists $\boldsymbol{y} \gg \boldsymbol{0}$ with $F(\boldsymbol{x}) \leq \boldsymbol{y}$ for all $\boldsymbol{x} \in \mathbb{R}_{+}^{n}$. As $F$ is continuous and $F(\boldsymbol{x})=\boldsymbol{A}(\boldsymbol{x}) \boldsymbol{x}$, it follows from Theorem A1 that there exists some $\boldsymbol{x} \in \mathbb{R}_{+}^{n} \backslash\{\boldsymbol{0}\}$ with $F(\boldsymbol{x})=\boldsymbol{x}$. As $F(\boldsymbol{x})$ is strictly positive for any non-zero $\boldsymbol{x}$, this implies that $\boldsymbol{x} \gg 0$.

Proof of Lemma 2: First note that $\boldsymbol{x}(0) \in \mathbb{R}_{+}^{n}$ implies $\boldsymbol{x}(t) \in \mathbb{R}_{+}^{n}$ for all $t \geq 0$. Thus the $l_{1}$ norm of $\boldsymbol{x}(t)$ is given by $\mathbf{1}^{T} \boldsymbol{x}(t)$. For any $t \geq 1, \boldsymbol{x}(t)=\boldsymbol{A}(\boldsymbol{x}(t-1)) \boldsymbol{x}(t-1)$ for some $\boldsymbol{x}(t-1) \in \mathbb{R}_{+}^{n}$. From the form of $\boldsymbol{A}(\boldsymbol{x})$, we can see that for $\boldsymbol{x}=\left(x_{1} x_{2}\right)^{T}$,

$$
\boldsymbol{1}^{T} \boldsymbol{A}(\boldsymbol{x})=\left(R_{1} F_{1} S_{1} e^{-\alpha x_{1}} \quad R_{2} F_{2} S_{2} e^{-\alpha x_{2}}\right)
$$

Hence

$$
|\boldsymbol{A}(\boldsymbol{x}) \boldsymbol{x}|_{1}=x_{1} R_{1} F_{1} S_{1} e^{-\alpha x_{1}}+x_{2} R_{2} F_{2} S_{2} e^{-\alpha x_{2}}
$$

As $x_{i} e^{-\alpha x_{i}} \leq(\alpha e)^{-1} \forall x_{i} \geq 0$, we have that 


$$
\begin{aligned}
|\boldsymbol{A}(\boldsymbol{x}) \boldsymbol{x}|_{1} & =x_{1} R_{1} F_{1} S_{1} e^{-\alpha x_{1}}+x_{2} R_{2} F_{2} S_{2} e^{-\alpha x_{2}} \\
& \leq(\alpha e)^{-1}\left(R_{1} F_{1} S_{1}+R_{2} F_{2} S_{2}\right)
\end{aligned}
$$

$\forall \boldsymbol{x} \geq 0$. As our solution $\boldsymbol{x}(t)$ is of the form $\boldsymbol{A}(\boldsymbol{x}) \boldsymbol{x}$ for any $t \geq 1$, this proves the lemma with $M=(\alpha e)^{-1}\left(R_{1} F_{1} S_{1}+R_{2} F_{2} S_{2}\right)$.

Proof of Theorem 1: $\boldsymbol{A}(\boldsymbol{x}) \boldsymbol{x} \gg \mathbf{0}$ by Lemma 1, so it is certainly true that $\mathbb{R}_{+}^{n} \backslash\{\mathbf{0}\}$ is forward invariant. As $d_{i}\left(x_{i}\right) \in(0,1)$ it can be seen that $\boldsymbol{F}^{\prime}(\mathbf{0})=\boldsymbol{A}(\mathbf{0})$ is irreducible for $\alpha>0, F_{i}>0, S_{i} \in(0,1)$ and $R_{i} \in(0,1), i=1,2 . \quad$ Also, $\quad \rho(\boldsymbol{A}(\mathbf{0}))>1$ implies that $\rho\left(\boldsymbol{A}(\mathbf{0})^{T}\right)>1$, so there exists some $\boldsymbol{v} \gg \mathbf{0}$ with $\boldsymbol{A}(\mathbf{0})^{T} \boldsymbol{v} \gg \boldsymbol{v}$ (just choose the eigenvector of $\boldsymbol{A}(\mathbf{0})^{T}$ corresponding to $\left.\rho\left(\boldsymbol{A}(\mathbf{0})^{T}\right)\right)$. This implies that we can choose $r_{0}>1$ with $\boldsymbol{A}(\mathbf{0})^{T} \boldsymbol{v} \geq r_{0} \boldsymbol{v}$. Finally, Lemma 2 implies that the system defined by $F(\boldsymbol{x})=\boldsymbol{A}(\boldsymbol{x}) \boldsymbol{x}$ is point dissipative. The result now follows immediately from Theorem A2.

Proof of Theorem 2: $F(\boldsymbol{x})=\boldsymbol{A}(\boldsymbol{x}) \boldsymbol{x}$ is strongly positive by Lemma 1 and $\mathbb{R}_{+}^{n} \backslash\{\boldsymbol{0}\}$ is forward invariant under $F$. It follows from Lemma 2 that the system (2) is point-dissipative. Note that $\boldsymbol{F}^{\prime}(\mathbf{0})=\boldsymbol{A}(\mathbf{0})$ is irreducible for $d\left(x_{i}\right) \in(0,1), \quad \alpha>0, F_{i}>0, S_{i} \in(0,1)$ and $R_{i} \in(0,1), i=1,2$. If $\rho(\boldsymbol{A}(\mathbf{0}))>1$, then $\rho\left(\boldsymbol{A}(\mathbf{0})^{T}\right)>1$ so there exists some $\boldsymbol{v} \gg \mathbf{0}$, $r_{0} \geq 1$ with $\boldsymbol{A}(\mathbf{0})^{T} \boldsymbol{v} \geq r_{0} \boldsymbol{v}$, as in the proof of Theorem 1 . As $F(\boldsymbol{x}) \gg \mathbf{0}$ for any non-zero $\boldsymbol{x}$, the result now follows immediately from Theorem A3 with $X_{0}=\{\mathbf{0}\}$. This implies patch-persistence when $\rho(\boldsymbol{A}(\mathbf{0}))>1$. 


\section{Appendix 3}

\section{Tables}

Table S1 Mean oviposition period \pm standard error (s.e.) in days, for each generation and host. Means followed by the same letter do not differ statistically.

\begin{tabular}{llll}
\hline Generation & Mean \pm s.e. & Host & Mean \pm s.e. \\
\hline F1 & $15.20 \pm 2.86$ a & Raspberry & $27.56 \pm 2.69 \mathrm{a}$ \\
& & & \\
F2 & $17.90 \pm 3.94 \mathrm{a}$ & Strawberry & $5.17 \pm 1.20 \mathrm{~b}$ \\
\hline
\end{tabular}

Table S2 Means \pm standard errors (s.e.) of the percentage of viable eggs for generations and hosts. Means followed by the same letter do not differ statistically.

\begin{tabular}{llll}
\hline Generation & Mean \pm s.e. & Host & Mean \pm s.e. \\
\hline F1 & $16.91 \pm 5.14 \mathrm{~b}$ & Raspberry & $21.60 \pm 3.49 \mathrm{~b}$ \\
& & & \\
F2 & $32.10 \pm 5.88 \mathrm{a}$ & Strawberry & $33.47 \pm 14.69 \mathrm{a}$ \\
\hline
\end{tabular}

Table S3 Mean macronutrient concentrations $(\mu \mathrm{g} / \mu \mathrm{L}) \pm$ standard errors for each generation.

\begin{tabular}{|c|c|c|c|c|}
\hline \multirow[t]{2}{*}{ Generation } & \multicolumn{4}{|l|}{ Macronutrient } \\
\hline & Lipid & Protein & Carbohydrate & Glycogen \\
\hline$F 1-$ raspberry & $10.43 \pm 0.1075 a$ & $0.13 \pm 0.0064 \mathrm{a}$ & $0.02 \pm 0.0066 \mathrm{a}$ & $0.03 \pm 0.0148 \mathrm{a}$ \\
\hline$F 2$ - raspberry & $10.37 \pm 0.0756 \mathrm{a}$ & $0.13 \pm 0.0061 \mathrm{a}$ & $0.02 \pm 0.0003 \mathrm{a}$ & $0.04 \pm 0.0074 \mathrm{a}$ \\
\hline F3 - raspberry & $10.27 \pm 0.0563 \mathrm{a}$ & $0.12 \pm 0.0049 \mathrm{a}$ & $0.02 \pm 0.0014 \mathrm{a}$ & $0.05 \pm 0.0123 \mathrm{a}$ \\
\hline F3 - strawberry & $10.43 \pm 0.0399 a$ & $0.14 \pm 0.0074 \mathrm{a}$ & $0.02 \pm 0.0020 \mathrm{a}$ & $0.03 \pm 0.0117 \mathrm{a}$ \\
\hline
\end{tabular}




\section{References}

Abraham, J., Zhang, A., Angeli, S., Abubeker, S., Michel, C., Feng, Y., \& Rodriguez-

Saona, C. 2015. Behavioral and Antennal Responses of Drosophila suzukii (Diptera:

Drosophilidae) to Volatiles from Fruit Extracts. Environmental Entomology, 44:356-367.

Alsefri, M., Sudell, M., García-Fiñana, M. et al. 2020. Bayesian joint modelling of longitudinal and time to event data: a methodological review. BMC Medical Research Methodology 20:94.

Atallah, J., Teixeira, L., Salazar, R., Zaragoza, G., \& Kopp, A. 2014. The making of a pest: the evolution of a fruit-penetrating ovipositor in Drosophila suzukii and related species. Proc. of the Royal Society B: Biological Sciences, 281(1781):20132840.

Belay, D.B., Kifle, Y.G., Goshu, A.T., et al. 2017. Joint Bayesian modeling of time to malaria and mosquito abundance in Ethiopia. BMC Infectious Diseases. 17(1):415.

Bernal, J.S. and Medina, R.F. 2018. Agriculture sows pests: How crop domestication, host shifts, and agricultural intensification can create insect pests from herbivores. Current opinion in insect science, 26:76-81.

Bing, X., Gerlach, J., Loeb, G., \& Buchon, N. 2018. Nutrient-dependent impact of microbes on Drosophila suzukii development. MBio, 9(2):e02199-17.

Bolda, M.P., Goodhue, R.E., \& Zalom, F.G. 2010. Spotted Wing Drosophila: Potential Economic Impact of a Newly Established Pest. Agricultural and Resource Economics Update, 13(3):5-8.

Burrack, H.J., Fernandez, G.E., Spivey, T., \& Kraus, D.A. 2013. Variation in selection and utilization of host crops in the field and laboratory by Drosophila suzukii Matsumara (Diptera: Drosophilidae), an invasive frugivore. Pest Management Science, 69(10):1173-1180.

Buruga, J.H. \& Olembo, R.J. 1971. Plant Food Preferences of Some Sympatric Drosophilids of Tropical Africa. Biotropica, 3(2):151-158.

Bryant, T.B., Dorman, S.J., Reisig, D.D., Dillard, D., Schürch, R. \& Taylor, S.V. 2020. Reevaluating the Economic Injury Level for Brown Stink Bug (Hemiptera: Pentatomidae) at Various Growth Stages of Maize. Journal of Economic 
Entomology, 113(5):2250-2258.

Calabria, G., Máca, J., Bächli, G., Serra, L., \& Pascual, M. 2012. First records of the potential pest species Drosophila suzukii (Diptera: Drosophilidae) in Europe. Journal of Applied Entomology, 136:139-147.

Cheng, C.Y., Chen, S.S., \& Zou, X. 2019. On an age structured population model with density-dependent dispersals between two patches. Mathematical Biosciences and Engineering, 16(5):4976-4998.

Chesson, P. 2012. Scale transition theory: its aims, motivations and predictions. Ecological Complexity, 10:52-68.

Cichón, L., Garrido, S., Lago, J. 2015. Primera detección de Drosophila suzukii (Matsumura, 1939) (Diptera: Drosophilidae) en frambuesas del Valle de Río Negro, Argentina. In: IX Congreso Argentino de Entomología Posadas: libro de resúmenes. Universidad Nacional de Misiones, Posadas.

Comins, H.N., Hassell, M.P. and May, R.M., 1992. The Spatial Dynamics of Host-Parasitoid Systems. Journal of Animal Ecology, pp.735-748.

Costa, M.I., Faria, L. D. B. 2010. Integrated pest management: theoretical insights from a threshold policy. Neotropical entomology, 39(1):1-8.

Costa, M.I.D.S., \& dos Anjos, L. 2018. Multiple hydra effect in a predator-prey model with Allee effect and mutual interference in the predator. Ecological modelling, 373:22-24.

Crava, C.M., Ramasamy, S., Ometto, L., Anfora, G., \& Rota-stabelli, O. 2016. Evolutionary Insights into Taste Perception of the Invasive Pest Drosophila suzukii. G3: Genes, Genomes, Genetics, 6:4185-4196.

Cushing, J.M. 1998. An introduction to structured population dynamics. Society for industrial and applied mathematics.

Demétrio, C.G.B., Hinde, J., Moral, R.A. 2014. Models for overdispersed data in Entomology. In: Ferreira, C.P., Godoy, W.A.C. (Eds.) Ecological Modelling Applied to Entomology, Springer.

Dennis, B., Desharnais, R.A., Cushing, J.M., Henson, S.M and Costantino, R.F. 2001. Estimating chaos and complex dynamics in an insect population. Ecological 
Monographs, 71(2):277-303.

Deprá, M., Poppe, J.L., Schmitz, H.J., De Toni, D.C., \& Valente, V.L. 2014. The first records of the invasive pest Drosophila suzukii in the South American continent. Journal of Pest Science 87:379-383.

Dercole. F, \& Rinaldi. S. 2012. Bifurcations. In: Hastings A \& Gross LJ. Encyclopedia of theoretical ecology.University of California Press, Berkeley.

Dey, S., Bose, J., \& Joshi, A. 2012. Adaptation to larval crowding in Drosophila ananassae leads to the evolution of population stability. Ecology and Evolution, 2(5):941-951.

Diepenbrock, L.M., Swoboda-Bhattarai, K.A., \& Burrack, H.J. 2016. Ovipositional preference, fidelity, and fitness of Drosophila suzukii in a co-occurring crop and non-crop host system. Journal of Pest Science, 89(3):761-769.

Djawdan, M., Sugiyama, T.T., Schlaeger, L.K., Bradley, T.J., \& Rose, M.R. 2004. Metabolic aspects of the trade-off between fecundity and longevity in Drosophila melanogaster. In Methuselah Flies: A Case Study in the Evolution of Aging (pp. 145-164).

Duelli, P. and Obrist, M.K. 2003. Regional biodiversity in an agricultural landscape: the contribution of seminatural habitat islands. Basic and applied ecology, 4(2):129-138.

Eager, E.A., Rebarber, R. and Tenhumberg, B. 2012. Choice of density-dependent seedling recruitment function affects predicted transient dynamics: a case study with Platte thistle. Theoretical ecology, 5(3):387-401.

Elaydi, S., 2005. An Introduction to Difference Equations, Undergraduate Texts in Mathematics. Springer New York.

Faria, L.D.B., Orsi, L., Trinca, L.A. \& Godoy, W.A.C. 1999. Larval predation by Chrysomya albiceps on Cochliomyia macellaria, Chrysomya megacephala and Chrysomya putoria. Entomologia Experimentalis et Applicata 90:149-155.

Geritz, S.A. and Kisdi, É., 2004. On the mechanistic underpinning of discrete-time population models with complex dynamics. Journal of Theoretical Biology, 228(2), pp.261-269.

González, G., Mary, A.L. \& Goñi, B. 2015. Drosophila suzukii (Matsumura) found in 
Uruguay. Drosophila Information Service 98(10):103-107.

Goodsman, D.W., Cooke, B.J. and Lewis, M.A. 2017. Positive and negative densitydependence and boom-bust dynamics in enemy-victim populations: a mountain pine beetle case study. Theoretical Ecology, 10(2):255-267.

Guidolin, A.S. \& Cônsoli, F.L. 2017. Symbiont Diversity of Aphis (Toxoptera) citricidus (Hemiptera: Aphididae) as Influenced by Host Plants. Microbial Ecology, 73(1):201-210.

Guiver, C., David, P., and Stuart, T. 2017. “A Necessary Condition for Dispersal Driven Growth of Populations with Discrete Patch Dynamics.” Journal of Theoretical Biology 424:11-25.

Gupta, J.P. 1970. Description of a new species of Phorticella and Zaprionus (Drosophilidae) from Índia. Proceedings of the Indian National Science Academy 36:62-70.

Hanski, I.A., \& Gaggiotti, O.E. (Eds.) 2004. Ecology, genetics and evolution of metapopulations. Academic Press.

Hardin, J.A., Kraus, D.A., \& Burrack, H.J. 2015. Diet quality mitigates intraspecific larval competition in Drosophila suzukii. Entomologia Experimentalis et Applicata, 156(1): 59-65.

Hastings, A., Abbott, K.C., Cuddington, K., Francis, T., Lai, Y.C., Morozov, A., Petrovskii, S. and Zeeman, M.L. 2021. Effects of stochasticity on the length and behaviour of ecological transients. bioRxiv.

Heaps, A.J., Dawson, T.D., Briggs, J.C., Hansen, M.A. \& Jensen, J.L. 2016. Deriving Population Growth Models by Growing Fruit Fly Colonies. The American Biology Teacher 78(3):221-225.

Heinrichs, J.A., Lawler, J.J. and Schumaker, N.H. 2016. Intrinsic and extrinsic drivers of source-sink dynamics. Ecology and evolution, 6(4):892-904.

Hengeveld, R. 1989. Dynamics of biological invasions. Springer Science \& Business Media.

James, P.M., Cooke, B., Brunet, B.M., Lumley, L.M., Sperling, F.A., Fortin, M.J., ... \& Sturtevant, B.R. 2015. Life-stage differences in spatial genetic structure in an irruptive forest insect: implications for dispersal and spatial synchrony. Molecular 
Ecology, 24(2):296-309.

Jansen, V.A. and Sigmund, K. 1998. Shaken, not stirred: On permanence in ecological communities.

Kaneshiro, K.Y. 1983 Drosophila (Sophophora) suzukii (Matsumura). In: Proceedings of the Hawaiian Entomological Society 24:179.

Kennedy, G.G. \& Storer, N.P. 2000. Lifesystems of polyphagous arthropod pests in temporally unstable cropping systems. Annual Review of Entomology. 45:467493.

Kinjo, H., Kunimi, Y., Ban, T., Nakai, M., Isaacs, R., Miller, J.C., ... Yukinari, M. 2013. Oviposition efficacy of Drosophila suzukii (Diptera: Drosophilidae) on different cultivars of blueberry. Journal of Economic Entomology, 106(4):1767-1771.

Klesener, D.F., dos Santos, R.S.S., Gebler, L., \& Marchioretto, L.D.R. 2018. Population fluctuation and infestation of Drosophila suzukii in berry crops in Southern Brazil. Embrapa Uva e Vinho-Artigo em periódico indexado (ALICE).

Klomp, H. 1964. Intraspecific competition and the regulation of insect numbers. Annual review of entomology, 9(1):17-40.

Knapp, L., Mazzi, D., \& Finger, R. 2021. The economic impact of Drosophila suzukii: perceived costs and revenue losses of Swiss cherry, plum and grape growers. Pest Management Science, 77(2):978-1000.

Langille, A.B., Arteca, E.M., Ryan, G.D., Emiljanowicz, L.M., \& Newman, J.A. 2016. North American invasion of Spotted-Wing Drosophila (Drosophila suzukii): a mechanistic model of population dynamics. Ecological Modelling, 336:70-81.

Lee, J.C., Bruck, D.J., Dreves, A.J., Ioriatti, C., Vogt, H., \& Baufeld, P. 2011a. In Focus: Spotted wing drosophila, Drosophila suzukii, across perspectives. Pest Management Science, 67(11):1349-1351.

Lee, J.C., Bruck, D.J, Curry, H., Edwards, D., Haviland, D.R., Steenwyk, A. Van, \& Yorgey B.M. 2011b. The susceptibility of small fruits and cherries to the spottedwing drosophila, Drosophila suzukii. Pest Management Science, 67:1358-1367.

Lee, K.P., Simpson, S.J., Clissold, F.J., Brooks, R., Ballard, J.W.O., Taylor, P.W., ... Raubenheimer, D. 2008. Lifespan and reproduction in Drosophila: New insights from nutritional geometry. Proceedings of the National Academy of Sciences of the 
United States of America, 105(7):2498-2503.

Leslie, P.H. 1945. On the use of matrices in certain population mathematics. Biometrika, 33(3):183-212.

Lorenz, M.W. 2003. Adipokinetic hormone inhibits the formation of energy stores and egg production in the cricket Gryllus bimaculatus. Comparative Biochemistry and Physiology Part B, 136(2):197-206.

Lucia Mary, A. \& Goñi, B. 2015 Drosophila suzukii (Matsumura) found in Uruguay. Drosophila Information Service, 98(10):67-75.

Luque, B., Lacasa, L., Ballesteros, F.J. \& Robledo, A. 2011. Feigenbaum Graphs: A Complex Network Perspective of Chaos. PLoS ONE 6(9):e22411.

Lotka, A.J. 1925. Elements of Physical Biology, William and Wilkins, Baltimore.

Malaquias, J.B., Godoy, W.A., Garcia, A.G., Ramalho, F.D.S., \& Omoto, C. 2017. Larval dispersal of Spodoptera frugiperda strains on Bt cotton: a model for understanding resistance evolution and consequences for its management. Scientific reports, $7(1): 1-10$.

Matavelli, C., Carvalho, M.J.A., Martins, N.E., \& Mirth, C.K. 2015. Differences in larval nutritional requirements and female oviposition preference reflect the order of fruit colonization of Zaprionus indianus and Drosophila simulans. Journal of Insect Physiology, 82:66-74.

Matsumura, S. 1931. 6000 illustrated insects of Japan-empire (in Japanese). Tokohshoin, Tokyo, 1497 pp.

Matzkin, L.M., Johnson, S., Paight, C., \& Markow, T.A. 2013. Preadult Parental Diet Affects Offspring Development and Metabolism in Drosophila melanogaster. PLoS ONE, 8(3):1-6.

May, R.M. 1995. Necessity and chance: deterministic chaos in ecology and evolution. arXiv preprint math/9507222.

Mazzi, D., \& Dorn, S. 2012. Movement of insect pests in agricultural landscapes. Annals of Applied Biology, 160(2):97-113.

McIntire, K.M., \& Juliano, S.A. 2018.How can mortality increase population size? A test of two mechanistic hypotheses. Ecology, 99(7):1660-1670.

Medina-Muñoz, M.C., Lucero, X., Severino, C., Cabrera, N., Olmedo, D., Pino, F. Del, 
... Godoy-Herrera, R. 2015. Drosophila suzukii arrived in Chile. Drosophila Information Service, 98(23):136-137.

Moral, R.A., Hinde, J., Demétrio, C.G.B. 2017. Half-normal plots and overdispersed models in R: the hnp package. Journal of Statistical Software 81(10):1-23.

Moretti, T.C., Coutinho, R.M., Moral, R.A. et al. 2013. Quantitative and qualitative dynamics of exotic and native blowflies (Diptera: Calliphoridae) with migrations among municipalities. Community Ecology 14:249-257.

Mueller, L.D., \& Ayala, F.J. 1981. Trade-off between r-selection and K-selection in Drosophila populations. Proceedings of the National Academy of Sciences, 78(2):1303-1305.

Nestel, D., Carvalho, J., \& Nemny-Lavy, E. 2004. The spatial dimension in the ecology of insect pests and its relevance to pest management. In Insect pest management (pp. 45-63). Springer, Berlin, Heidelberg.

Neubert, M.G., Caswell, H. and Murray, J.D. 2002. Transient dynamics and pattern formation: reactivity is necessary for Turing instabilities. Mathematical biosciences, 175(1):1-11.

Obadia, B., Keebaugh, E.S., Yamada, R., Ludington, W.B., \& Ja, W.W. 2018. Diet influences host - microbiota associations in Drosophila. Proceedings of the National Academy of Sciences of the United States of America, 115(20):4547-4548.

Okubo, A., \& Levin, S.A. 2001. The basics of diffusion. In Diffusion and ecological problems: Modern perspectives (pp. 10-30). Springer, New York, NY.

Olazcuaga, L., Rode, N.O., Foucaud, J., Facon, B., Ravigné, V., Ausset, A., ... \& Hufbauer, R.A. 2019. Oviposition preference and larval performance of Drosophila suzukii (Diptera: Drosophilidae), spotted-wing drosophila: Effects of fruit identity and composition. Environmental entomology, 48(4):867-881.

Ørsted, I.V., \& Ørsted, M. 2019. Species distribution models of the Spotted Wing Drosophila (Drosophila suzukii, Diptera: Drosophilidae) in its native and invasive range reveal an ecological niche shift. Journal of Applied Ecology, 56(2):423-435.

Pal, N., Samanta, S., Martcheva, M., \& Chattopadhyay, J. 2018. Role of bi-directional migration in two similar types of ecosystems. Mathematics, 6(3):36.

Poyet, M., Le Roux, V., Gibert, P., Meirland, A., Prévost, G., Eslin, P., \& Chabrerie, O. 
2015. The wide potential trophic niche of the Asiatic fruit fly Drosophila suzukii: The key of its invasion success in temperate Europe? PLoS ONE, 10(11).

Powell, J.A. and Bentz, B.J. 2014. Phenology and density-dependent dispersal predict patterns of mountain pine beetle (Dendroctonus ponderosae) impact. Ecological Modelling, 273:173-185.

Prout, T. \& Mcchesney, F. 1985. Competition among immatures affects their adult fertility: population dynamics. American Naturalist, 126:521-558.

Rand, T.A., Tylianakis, J.M., \& Tscharntke T (2006) Spillover edge effects: the dispersal of agriculturally subsidized insect natural enemies into adjacent natural habitats. Ecology letters, 9(5), 603-614. Doi: https://doi.org/10.1111/j.14610248.2006.00911.x

Raubenheimer, D. \& Simpson, S.J. 2018. Nutritional ecology and foraging theory. Current Opinion in Insect Science, 27:1-8.

Reigada, C., Schreiber, S.J., Altermatt, F. \& Holyoak, M. 2015. Metapopulation dynamics of ephemeral patches. The America Naturalist, 185.

Reigada, C., de Andrade Moral. R., Demétrio, C.G.B., \& Parra, J.R.P. 2018. Cross-crop effects on larval growth, survivorship and fecundity of Helicoverpa armigera. Journal of pest science, 91(1):121-131.

Reyes, J.A., \& Lira-Noriega, A. 2020. Current and future global potential distribution of the fruit fly Drosophila suzukii (Diptera: Drosophilidae). The Canadian Entomologist, 152(4):587-599.

Rodrigues, M.A., Martins, N.E., Balancé, L.F., Broom, L.N., Dias, A.J.S., Fernandes, A.S.D., ...Mirth, C.K. 2015. Drosophila melanogaster larvae make nutritional choices that minimize developmental time. Journal of Insect Physiology, 81:69-80.

Roe, J.H. 1955. The determination of sugar in blood and spinal fluid with anthrone reagent. Journal of Biological Chemistry, 212:335-343.

Ricker, W.E. 1954. Stock and recruitment. Journal of the Fisheries Board of Canada, 11(5): 559-623.

Rinnan, D.S. 2018. The dispersal success and persistence of populations with asymmetric dispersal. Theoretical Ecology, 11(1):55-69.

Rizopoulos, D.J.M. 2010. An R package for the joint modelling of longitudinal and time- 
to-event data. Journal of Statistical Software, 35(9):1-33.

Ruxton, G.D. 1996. Density-dependent migration and stability in a system of linked populations. Bulletin of Mathematical Biology, 58(4):643-660.

SENASICA, 2013. Mosca del vinagre de alas manchadas (Drosophila suzukii

Mastsumura, 1931). Dirección General de Sanidad Vegetal-Sistema Nacional de Vigilancia Epidemiolófica Fitosanitaria. México D.F., Ficha Técnica No 7, 22 pp.

Shin, S.C., Kim, S.H., You, H., Kim, B., Kim, A.C., Lee, K.A., .. Lee, W.J. 2011.

Drosophila Microbiome Modulates Host Developmental and Metabolic

Homeostasis via Insulin Signaling. Science, 334(6056):670-674.

Smith, H.L., \& Thieme, H.R. 2011. Dynamical systems and population persistence (Vol. 118). American Mathematical Soc.

Sommer, A.J. \& Newell, P.D. 2018. Metabolic Basis for Mutualism Between Gut Bacteria and Its Impact on Their Host Drosophila melanogaster. Applied and Environmental Microbiology, 85(2).

Stearns, A.S.C. 1989. Trade-Offs in Life-History Evolution. British Ecological Society, 3(3): 259-268.

Su, Q., Zhou, X., \& Zhang, Y. 2013. Symbiont-mediated functions in insect hosts.

Communicative and Integrative Biology, 6(3):1-7.

Tung, S., Rajamani, M., Joshi, A., \& Dey, S. 2019. Complex interaction of resource availability, life-history and demography determines the dynamics and stability of stage-structured populations. Journal of theoretical biology, 460:1-12

Van Driesche, R.G. \& Murray, T.J. 2004. Overview of Testing Schemes and Designs Used to Estimate Host Ranges. Assessing Host Ranges for Parasitoids and Predators used in Classical Biological Control: A Guide to Best Practice (ed. by RG Van Driesche \& R Reardon), USDA Forest Service, Morgantown, West Virginia, USA, pp. $68-89$.

Van Handel, E. 1985. Rapid determination of total lipids in mosquitoes. Journal of the American Mosquito Control Association, 1(3):302-304.

Van Voorn, G.A., \& Kooi, B.W. 2017. Combining bifurcation and sensitivity analysis for ecological models. The European Physical Journal Special Topics, 226(9):21012118. 
Vortkamp, I., Schreiber, S.J., Hastings, A. and Hilker, F.M., 2020. Multiple attractors and long transients in spatially structured populations with an Allee effect. Bulletin of mathematical biology, 82(6):1-21.

Walsh, D.B., Bolda, M.P., Goodhue, R.E., Dreves, A.J., Lee, J., Bruck, D.J., ... Zalom, F.G. 2011. Drosophila suzukii (Diptera: Drosophilidae): Invasive Pest of Ripening Soft Fruit Expanding its Geographic Range and Damage Potential. Journal of Integrated Pest Management, 2(1):G1-G7.

Winkler, A., Jung, J., Kleinhenz, B., \& Racca, P. 2020. A review on temperature and humidity effects on Drosophila suzukii population dynamics. Agricultural and Forest Entomology, 22(3):179-192.

Wieser, A., Reuss, F., Niamir, A., Müller, R., O’Hara, R.B., \& Pfenninger, M. 2019. Modelling seasonal dynamics, population stability, and pest control in Aedes japonicus japonicus (Diptera: Culicidae). Parasites \& vectors, 12(1):1-12.

Wong, J.S., Wallingford, A.K., Loeb, G.M., \& Lee, J.C. 2018. Physiological status of Drosophila suzukii (Diptera: Drosophilidae) affects their response to attractive odours. Journal of Applied Entomology, 142(5):473-482.

Yun, J., Roh, W., Whon, W., Jung, M., Kim, M., Park, D., \& Yoon, C. 2014. Insect Gut Bacterial Diversity Determined by Environmental Habitat, Diet, Developmental Stage, and Phylogeny of Host. Applied and Environmental Microbiology, 80(17):5254-5264.

Zhang, H, Garratt, M.P., Bailey, A., Potts, S.G., \& Breeze, T. 2018. Economic valuation of natural pest control of the summer grain aphid in wheat in South East England. Ecosystem Services, 30:149-157.

Zhou, Y., \& Kot, M. 2011. Discrete-time growth-dispersal models with shifting species ranges. Theoretical Ecology, 4(1):13-25. 